\title{
Anosov diffeomorphisms, anisotropic BV spaces and regularity of foliations
}

\author{
WAEL BAHSOUN $\dagger$ and CARLANGELO LIVERANI $\ddagger$ \\ $\dagger$ Department of Mathematical Sciences, Loughborough University, \\ Loughborough, Leicestershire, LE11 3TU, UK \\ (e-mail:W.Bahsoun@lboro.ac.uk) \\ $\ddagger$ Dipartimento di Matematica, Università di Roma II, Tor Vergata, \\ Via della Ricerca Scientifica, 00133 Roma, Italy \\ (e-mail: liverani@mat.uniroma2.it)
}

(Received 15 March 2020 and accepted in revised form 1 April 2021)

\begin{abstract}
Given any smooth Anosov map, we construct a Banach space on which the associated transfer operator is quasi-compact. The peculiarity of such a space is that, in the case of expanding maps, it reduces exactly to the usual space of functions of bounded variation which has proved to be particularly successful in studying the statistical properties of piecewise expanding maps. Our approach is based on a new method of studying the absolute continuity of foliations, which provides new information that could prove useful in treating hyperbolic systems with singularities.
\end{abstract}

Key words: anisotropic Banach spaces, Anosov diffeomorphsims, foliation regularity, transfer operators.

2020 Mathematics Subject Classification: 37A25 (Primary); 37A30, 37D20 (Secondary)

\section{Introduction}

Starting with the paper [BKL], there has been a growing interest in the possibility of developing a functional analytic setting allowing the direct study of the transfer operator of a hyperbolic dynamical system. The papers [GL, GL1, BT, BT1, B1, B2, B3, B4, T1] have now produced quite satisfactory results for the case of Anosov diffeomorphisms (or, more generally, for uniformly hyperbolic basic sets). Although the theory is not yet complete, important results, have been obtained for flows [L, BuL, BuL2, GLP, FT2, DyZ, D17], group extensions and skew products [F11, AGT]. Moreover, recently, a strong relationship with techniques used in semiclassical analysis (see, e.g. [FR, FRS, FT1, FT2, DyZ]) has been unveiled. Also, one should mention the recent discovery of a deep relationship with the theory of renormalization of parabolic systems [GL19]. In addition, such an 
approach has proved to be very effective in the study of perturbations of dynamical systems [KL1, KL3] and in the investigation of limit theorems [G10]. At the same time, [KL2, KL4] have shown that this strategy can be extended to a large class of infinite-dimensional systems (coupled map lattices), but is limited to the case of piecewise expanding maps. However, there has been no progress in applying it to coupled lattices of Anosov maps when the coupling introduces discontinuities in the system. (The only available results are restricted to a special class of interactions that salvage structural stability [PS].) Moreover, only partial progress has been accomplished in extending such an approach to partially hyperbolic maps [T2] and to piecewise smooth uniformly hyperbolic systems [DL, BG1, BG2, DZ1, DZ2, DZ3, BaL, BDL]. The recent book [B5] provides an extensive account and a thorough illustration of the topic.

The present paper is motivated by the current shortcomings in the applications of the functional analytic strategy to piecewise smooth hyperbolic maps. Indeed, while in two dimensions the approach can be applied to a large class of systems [DZ2, DZ3], in higher dimensions it is limited to the case in which the map is well behaved up to and including the boundary [BG1, BG2] or some special skew product cases [Ga18, GL18]. In the case of piecewise expanding maps, the latter problems are dealt with by using different Banach spaces. In particular, a huge class of piecewise expanding maps can be treated by using the space of functions of bounded variation (BV) or their straightforward generalizations [Sa00, Li13a, Bu13, Li13b]. It is thus natural to construct Banach spaces that generalize BV and are adapted to the study of the transfer operator associated with hyperbolic maps.

BV-like spaces could allow one to extend the known results to higher-dimensional, possibly infinite-dimensional (coupled Anosov map lattices) systems. Also, they could allow one to treat higher-dimensional hyperbolic maps with strong singularities (e.g. billiards). In addition, such spaces should be useful for investigating numerically the spectrum of the transfer operator via Ulam-type perturbations, which proved to be very successful when dealing with expanding maps and BV functions [Liv]. Indeed, previous investigations of Ulam approximations for Anosov systems left several questions unanswered due to the inadequacy of the Banach spaces used (see, e.g., [BKL]).

Unfortunately, none of the Banach spaces proposed in the literature for the study of the transfer operator associated with general Anosov diffeomorphisms, or general piecewise Anosov, reduces exactly to BV when the stable direction is absent.

The purpose of this paper is to correct this state of affairs by introducing a template for Banach spaces with the above property. We apply it to the case of smooth Anosov diffeomorphisms. Although, for such examples, this provides limited new information, it shows that the proposed space is well adapted to the hyperbolic structure, and hence there is a concrete hope that this space can be adapted to study general piecewise Anosov maps and Anosov coupled map lattices in a unified setting. A substantial amount of work is still needed to find out whether or not such a hope has some substance. Nevertheless, the present arguments are worth presenting since they are remarkably simple and natural.

An additional fact of interest in the present paper is the characterization of invariant foliations and, more generally, the method used to study the evolution of foliations under the dynamics. It is well known that the stable foliation is only Hölder, although the leaves of 
the foliations enjoy the same regularity as the map. Nevertheless, a fundamental discovery by Anosov is that the holonomy associated to the foliation is absolutely continuous and the Jacobian is Hölder. The establishing of this fact is not trivial and, especially in the discontinuous case, entails a huge amount of work [KS]. Here we show that the properties of such foliations can be characterized infinitesimally, which considerably simplifies their description (see Definition 2.5). In particular, given a foliation $F$, the Jacobian $J^{F}$ of the associated holonomy can be seen as a quantity produced by a flow, of which we control the generator $H^{F}$. See Lemma B.7 for a precise explanation of this fact. We believe this point of view will be instrumental in treating discontinuous maps.

The structure of the paper is as follows. Section 2 contains the definition of the Banach space and the statement of the main theorem (Theorem 2.1). Section 3 contains the usual Lasota-Yorke estimate, while $\$ 4$ contains the estimate on the essential spectrum of the operator. Section 5 contains some comments on the peripheral spectrum. Appendix A reminds the reader of some convenient properties of $\mathcal{C}^{r}$ norms. Appendix B establishes various properties of the foliations of Anosov maps that should be folklore among experts, but we could not locate anywhere in the literature (in particular, the smoothness of the Jacobian of the stable holonomy along stable leaves). Moreover, as previously mentioned, such properties are expressed totally in local terms, contrary to the usual approach. Finally, Appendix $\mathrm{C}$ contains a few technical estimates on the test functions.

1.1. Notation. In this paper, we will use $C_{\sharp}$ to designate a constant that depends only on the map $T$ and on the choice of coordinates, but whose actual value is irrelevant to the tasks at hand. Hence, the value of $C_{\sharp}$ can change from one occurrence to the next and it is determined by the equation in which it appears. Analogously, we will use $C_{a, b, \ldots}, c_{a, b, \ldots}$ for generic constants that depend also on the quantities $a, b, \ldots$

\section{The Banach space}

Our goal is to develop a space in the spirit of BV for the study of the statistical properties of a dynamical system $(M, T, \mu)$, where $M$ is a compact $\mathcal{C}^{r}$ manifold, $T$ is uniformly hyperbolic and $\mu$ is the Sinai-Ruelle-Bowen (SRB) measure. (Of course, there are many other functional spaces to analyze such maps (e.g., see [B5]). However, we restrict to this class of maps to illustrate the construction of the space in the simplest possible form.) Let us be more precise.

2.1. The phase space. Let $r \geq 2$ be an integer and let $M$ be a $\mathcal{C}^{r} d$-dimensional compact manifold where the differentiable structure is the one induced by the atlas $\left\{V_{i}, \phi_{i}\right\}_{i=1}^{S}, V_{i} \subset$ $M, S \in \mathbb{N}$. More precisely, we consider a fixed smooth partition of unity $\left\{\vartheta_{i}\right\}$ subordinated to the cover $\left\{V_{i}\right\}$. We then define a smooth volume form $\omega$ by

$$
\int_{M} h d \omega=\sum_{i=1}^{S} \int_{U_{i}} h \circ \phi_{i}^{-1}(z) \vartheta_{i} \circ \phi_{i}^{-1}(z) d z,
$$

where $U_{i}:=\phi_{i}\left(V_{i}\right)$. From now on, all integrals will be with respect to such a form although we will not specify it explicitly. 
2.2. The map and the cones. We consider an Anosov diffeomorphism $T \in \operatorname{Diff}^{r}(M)$. That is, there exists $\lambda>1, v \in(0,1), c_{0} \in(0,1)$ and a continuous cone field (stable cone) $\mathcal{C}=\{C(\xi)\}_{\xi \in M}, \overline{C(\xi)}=C(\xi) \subset T_{\xi} M$ such that $D_{\xi} T^{-1} C(\xi) \subset \operatorname{int}\left(C\left(T^{-1}(\xi)\right)\right) \cup$ $\{0\}$ and (here the norm is defined by some smooth Riemannian structure, and the actual choice of such a structure will be irrelevant in the following; it will just affect the constants)

$$
\begin{aligned}
& \inf _{\xi \in M} \inf _{v \in C(\xi)}\left\|D_{\xi} T^{-n} v\right\|>c_{0} v^{-n}\|v\|, \\
& \inf _{\xi \in M} \inf _{v \notin C(\xi)}\left\|D_{\xi} T^{n} v\right\|>c_{0} \lambda^{n}\|v\| .
\end{aligned}
$$

In higher dimensions, a cone may have many geometric shapes. It is convenient, and useful, to ask that they be subsets $\mathcal{K}$ of the Grassmannian. More precisely, we can assume, without loss of generality, that, for each $\xi \in V_{i}$ and calling $\mathcal{M}\left(d_{u}, d_{s}\right)$ the set of $d_{u} \times d_{s}$ matrices,

$$
\begin{aligned}
\mathcal{K}_{\theta} & =\left\{U \in \mathcal{M}\left(d_{u}, d_{s}\right):\|U\| \leq \theta\right\}, \\
D \phi_{i} C(\xi) & =\left\{(x, y) \in \mathbb{R}^{d_{u}} \times \mathbb{R}^{d_{s}}=\mathbb{R}^{d}: x=U y, U \in \mathcal{K}_{1}\right\} \\
& =\left\{(x, y) \in \mathbb{R}^{d_{u}} \times \mathbb{R}^{d_{s}}=\mathbb{R}^{d}:\|x\| \leq\|y\|\right\},
\end{aligned}
$$

where $U$ is any $d_{u} \times d_{s}$ matrix. Then the strict cone field invariance reduces to the existence of $\eta \in(0,1)$ such that

$$
\begin{aligned}
& D \phi_{j} D T^{-1} C(\xi) \subset\left\{(x, y) \in \mathbb{R}^{d_{u}} \times \mathbb{R}^{d_{s}}=\mathbb{R}^{d}:\|x\| \leq \eta\|y\|\right\}, \\
& D \phi_{j^{\prime}} D T C_{c}(\xi) \subset\left\{(x, y) \in \mathbb{R}^{d_{u}} \times \mathbb{R}^{d_{s}}=\mathbb{R}^{d}:\|y\| \leq \eta\|x\|\right\},
\end{aligned}
$$

where $V_{j} \ni T^{-1}(\xi), V_{j^{\prime}} \ni T(\xi)$ and $C_{c}(\xi)=\overline{T_{\xi} M \backslash C(\xi)}$.

2.3. Transfer operator. We are interested in studying the statistical properties of the above systems. One key tool used to such an end is the transfer operator: for each $h \in \mathcal{C}^{1}$ we define (by det we mean the density of $T^{*} \omega$ with respect to $\omega$ )

$$
\mathcal{L} h=\left[h \cdot|\operatorname{det}(D T)|^{-1}\right] \circ T^{-1} .
$$

Accordingly, for each $n \in \mathbb{N}$,

$$
\int_{M} \varphi \mathcal{L}^{n} h=\int_{M} h \varphi \circ T^{n} .
$$

It is then clear that the behaviour of the integrals on the left-hand side of the above equation can be studied if one understands the spectrum of $\mathcal{L}$. Obviously, such a spectrum depends on the space on which the operator is defined. Several proposals have been developed to have spaces on which $\mathcal{L}$ is quasi-compact. Such proposals are extremely effective when the map is smooth (see [B5] for a review), but are less so for discontinuous systems. Since, in the case of expanding maps, BV is very effective [Li13b], it is natural to investigate whether one can construct a space, suitable for the study of invertible maps, that reduces to $\mathrm{BV}$ when the stable direction is absent. In the following sections, we define Banach spaces $\mathcal{B}^{0, q}$ and $\mathcal{B}^{1, q}$ that, when the stable direction is absent, reduce to $L^{1}$ and $\mathrm{BV}$, respectively (see Remark 2.18). Although we do not discuss discontinuous maps, this is certainly a first 
step towards develop a viable alternative to the current approaches. To show that the space is potentially well behaved, we prove the following theorem.

THEOREM 2.1. For each $q \in\{1, \ldots, r-2\}$ (note that this condition is non-vacuous only if $r \geq 3$; however, we stress that all the results related to the regularity of foliations in Appendix $B$ hold true for $r \geq 2$ ), the operator $\mathcal{L}$ has an extension (we still call such an extension $\mathcal{L})$, which belongs to $L\left(\mathcal{B}^{0, q}, \mathcal{B}^{0, q}\right)$ and $L\left(\mathcal{B}^{1, q}, \mathcal{B}^{1, q}\right)$. Moreover:

(1) $\mathcal{L}: \mathcal{B}^{1, q} \rightarrow \mathcal{B}^{1, q}$ is a quasi-compact operator with spectral radius 1 and essential spectral radius $\sigma_{\text {ess }}:=\max \left\{\lambda^{-1}, \nu\right\}$;

(2) the peripheral spectrum of $\mathcal{L}$ consists of finitely many finite groups; in particular, 1 is an eigenvalue; and

(3) setting $h_{*}:=\Pi_{1} 1$, where $\Pi_{1}$ is the spectral projection of $\mathcal{L}$ associated with the eigenvalue $1, h_{*}$ is canonically associated to a measure whose ergodic decomposition corresponds to the spectral decomposition for the Anosov map and consists of the physical measures.

Proof. The proof of (1) can be found in Lemma 4.1. The proof of (2) is given in Lemma 5.1. The canonical correspondence of $h_{*}$ with the distribution mentioned in point (3) is detailed in Lemma 2.16 (see also Remark 2.17 for the use of such a canonical correspondence in this paper) while, in Lemma 5.1, it is shown that the associated distribution is, in fact, a measure. Finally, the proof of (3) is provided by Lemma 5.2.

Observe that $B V \subset \mathcal{B}^{1, q}$ (see Remark 2.19), and hence the above theorem implies that, for Anosov maps, the spectrum $\sigma_{\mathcal{B}^{1, q}}(\mathcal{L})$ determines the decay of correlation for BV densities. In particular, consider a transitive Anosov map. Then $h_{*}$ is ergodic and corresponds to the unique $S R B$ measure $\mu_{S R B}$. Also, Theorem 2.1 implies that, for all $\theta>\sigma_{\text {ess }}$, there exists a constant $C_{\theta}>0$, finitely many eigenvalues $\left\{\theta_{j}\right\},\left|\theta_{j}\right| \in(\theta, 1)$, and finite rank operators $B_{j}: \mathcal{B}^{1,1} \rightarrow \mathcal{B}^{1,1}$, with spectral radius equal to one, such that, for all $\varphi \in \mathcal{C}^{2}$ and rectifiable sets $A$ (by rectifiable we mean that $\mathbb{1}_{A} \in B V$ ),

$$
\left|\int_{M} \varphi \circ T^{n} \mathbb{1}_{A}-\int_{M} \varphi \mu_{S R B} \int_{M} \mathbb{1}_{A}-\sum_{j} \theta_{j}^{n} \int_{M} \varphi B_{j}^{n} \mathbb{1}_{A}\right| \leq C\|\varphi\|_{\mathcal{C}^{2}}\left\|\mathbb{1}_{A}\right\|_{B V} \theta^{n}
$$

Note that a similar estimate could be obtained using the spectral properties on spaces already existing in the literature and by deducing the behaviour for BV densities using an approximation argument. However, this would produce a less sharp result (in particular, it would allow only $\theta>\sigma_{\text {ess }}^{\alpha}$ for some $\alpha<1$ ). In addition, the following are direct consequences of Theorem 2.1.

- The central limit theorem and other statistical properties for observables that are multipliers of BV hold via the usual spectral approach of analytic perturbation theory (see, e.g., [G15].

- Statistical aspects of random perturbations: let $T_{0}$ be a transitive Anosov map. Let $B_{T_{0}}$ be a sufficiently small neighborhood of $T_{0}$ in the $C^{1}$-topology so that condition (2.2) is satisfied for all $T \in B_{T_{0}}$ with uniform constants. Let $\Xi:=\sup _{l} \sum_{k}\left\|\left[\partial_{l}\left(D T_{0}^{-1}\right)_{l, k}\right]\right\|_{\mathcal{C}^{1}}$ 
and define the following family of maps

$$
G_{\Xi}=\left\{T \in C^{2}(M): T \in B_{T_{0}} \text { and } \sup _{l} \sum_{k}\left\|\left[\partial_{l}\left(D T^{-1}\right)_{l, k}\right]\right\|_{\mathcal{C}^{1}} \leq 2 \Xi\right\} .
$$

One can study, for instance, independent and identically distributed compositions with respect to some product probability measure $\mathbb{P}$ defined on $G_{\Xi}^{\mathbb{N}}$. Spectral properties of the annealed transfer operator associated with the above random map follows from this work and stability results can be obtained using the current setting and the framework of [KL1].

2.4. Foliations. A fundamental ingredient in the understanding of hyperbolic maps is the study of dynamical foliations. Hence a small digression is in order.

Definition 2.2. A $\mathcal{C}^{r} t$-dimensional foliation $W$ is a collection $\left\{W_{\alpha}\right\}_{\alpha \in A}$, for some set $A$, such that the $W_{\alpha}$ are pairwise disjoint, $\cup_{\alpha \in A} W_{\alpha}=M$ and, for each $\xi \in W_{\alpha}$, there exists a neighborhood $B(\xi)$ such that the connected component of $W_{\alpha} \cap B(\xi)$ containing $\xi$, call it $W(\xi)$, is a $\mathcal{C}^{r} t$-dimensional open submanifold of $M$. We will call $\mathcal{F}^{r}$ the set of $\mathcal{C}^{r} d_{s}$-dimensional foliations.

Definition 2.3. A foliation $W$ is adapted to the cone field $\mathcal{C}$ if, for each $\xi \in M, T_{\xi} W(\xi) \subset$ $C(\xi)$. Let $\mathcal{F}_{\mathcal{C}}^{r}$ be the set of $\mathcal{C}^{r} d_{s}$-dimensional foliations adapted to $\mathcal{C}$.

Given a $d_{s}$-foliation adapted to $\mathcal{C}$, we can associate to it local coordinates as follows. Let $\delta_{0}>0$ be sufficiently small so that, for each $\xi \in M$, there exists a chart $\left(V_{i}, \phi_{i}\right)$ with $\xi \in V_{i}$ and such that $U_{i}:=\phi_{i}\left(V_{i}\right)$ contains the ball $B_{\delta_{0}}\left(\phi_{i}(\xi)\right)$. (Here, and in the following, we use $B_{\delta}(x)$ to designate $\left\{z \in \mathbb{R}^{d^{\prime}}:\|x-z\| \leq \delta\right\}$ for any $d^{\prime} \in \mathbb{N}$.) Also, choose $U^{0}=$ $U_{u}^{0} \times U_{s}^{0} \subset \mathbb{R}^{d_{u}} \times \mathbb{R}^{d_{s}}$ with $U_{u}^{0}=B_{\delta_{0} / 2}(0), U_{s}^{0}=B_{\delta_{0} / 2}(0)$. Next, for each $z \in U_{i}$, let $W(z)$ be the connected component of $\phi_{i}(W)$ containing $z$. (Refer to Definition 2.2 for the exact meaning of 'connected component'. Also note the abuse of notation since we use the same name for the sub-manifold in $M$ and its image in the chart.) Define the function $F_{\xi}$ : $U^{0} \rightarrow \mathbb{R}^{d_{u}}$ by $\left\{\left(F_{\xi}(x, y)+x_{\xi}, y_{\xi}+y\right)\right\}=\left\{\left(w, y+y_{\xi}\right)\right\}_{w \in \mathbb{R}^{d_{u}}} \cap W\left(x+x_{\xi}, y_{\xi}\right)$, where $\left(x_{\xi}, y_{\xi}\right)=\phi_{i}(\xi)$. (The fact that the intersection is non-void and consists of exactly one point follows trivially from the fact that the foliation is adapted to the cone field. Hence the two manifolds are transversal.) That is, $W\left(x+x_{\xi}, y_{\xi}\right)$ is exactly the graph of the function $F_{\xi}(x, \cdot)+x_{\xi} \cdot$ Moreover,

$$
F_{\xi}(x, 0)=x
$$

In addition, we ask $\delta_{0}$ to be small enough for the expression of $D T$ in the above charts to be roughly constant. See Lemma B.5 and its proof for the precise condition.

Remark 2.4. The above construction defines the triangular coordinates $\mathbb{F}_{\xi}(x, y)=$ $\left(F_{\xi}(x, y), y\right)$, which describe locally the foliation. In fact, $\left(\phi_{i}^{-1} \circ \mathbb{F}_{\xi}\left(U^{0}\right), \mathbb{F}_{\xi}^{-1} \circ \phi_{i}\right)$ is a local chart of $M$ in which the foliation is trivial (the leaves are all parallel). In the following, we will often use such coordinates without mention if it will not create 
confusion. Also, to ease notation, we will confuse $V_{i}$ with $\phi_{i}\left(V_{i}\right)$ when not ambiguous. In addition, we will use $\mathbb{F}$ to indicate the collection of maps $\left\{\mathbb{F}_{\xi}\right\}$ and also for $F$. Of course, $\mathbb{F}$ is not unique, since we can chose different charts for the same $\xi$. However, different choices are equivalent so we assume that some choice has been made. Clearly, $\mathbb{F}$ uniquely defines $W$.

Definition 2.5. For each $r \in \mathbb{N}, r \geq 2$ and $L>0$, let

$$
\begin{aligned}
\overline{\mathcal{F}}_{\mathcal{C}}^{r}:=\left\{W \in \mathcal{F}_{\mathcal{C}}^{r}: \mathbb{F} \in \mathcal{C}^{r}\left(U^{0}, \mathbb{R}^{d}\right)\right\}, \\
\mathcal{W}_{L}^{r}:=\left\{W \in \overline{\mathcal{F}}_{\mathcal{C}}^{r}: \sup _{\xi} \sup _{x \in U_{u}^{0}|\alpha|=k} \sup _{|\alpha|=k}\left\|\partial_{y}^{\alpha} F_{\xi}(x, \cdot)\right\|_{\mathcal{C}^{0}\left(U_{s}^{0}, \mathbb{R}^{d_{u}}\right)} \leq L^{(k-1)^{2}}, 2 \leq k \leq r ;\right. \\
\left.\quad \sup _{\xi} \sup _{x \in U_{u}^{0}|\alpha|=k} \sup _{|\alpha|}\left\|\partial_{y}^{\alpha} H^{F_{\xi}}(x, \cdot)\right\|_{\mathcal{C}^{0}\left(U_{s}^{0}, \mathbb{R}^{d_{s}}\right)} \leq L^{(k+1)^{2}}, 0 \leq k \leq r-2\right\},
\end{aligned}
$$

where

$$
\begin{aligned}
H^{F_{\xi}}(x, y) & =\sum_{j=1}^{d_{u}}\left[\partial_{x_{j}}\left(\left[\partial_{y}\left(F_{\xi}\right)_{j}\right] \circ \mathbb{F}_{\xi}^{-1}\right)\right] \circ \mathbb{F}_{\xi}(x, y) \\
& =\sum_{i j} \partial_{x_{i}} \partial_{y}\left(F_{\xi}\right)_{j} \cdot\left(\partial_{x} F_{\xi}\right)_{i j}^{-1} .
\end{aligned}
$$

(Note that equation (2.3), Definition 2.3 and the subsequent description imply that $\left\|\partial_{y} F_{\xi}(x, y)\right\| \leq 1$. Hence, recalling (2.5), $\left\|F_{\xi}\right\|_{\infty} \leq \delta_{0}$. Accordingly, in the first line of the definition of $\mathcal{W}_{L}^{r}$, the cases $k \in\{0,1\}$ are superfluous.)

Remark 2.6. Since the invariant foliation is not $\mathcal{C}^{r}$ (in general, it is only Hölder, although it consists of $\mathcal{C}^{r}$ leaves) it does not belong to $\mathcal{W}_{L}^{r}$ for any $L$. However, it belongs to its closure if $L$ is large enough (see Remark B.3).

Remark 2.7. Note that the functions $H^{F}$ are related to the Jacobian of the stable holonomy (see Lemma B.7), and hence it does not make sense to require them to be uniformly smooth. In general, it is possible to control effectively only their Hölder norm, yet, restricted to the stable direction, they turn out to be smooth. Indeed, this is the whole content of Appendix B.

Remark 2.8. The role of $H^{F}$ in the definition of $\mathcal{W}_{L}^{r}$ will become apparent in the proof of the Lasota-Yorke inequality in Proposition 3.2, in particular, in (3.8). Hence, controlling the $\sup _{\xi} \sup _{x \in U_{u}^{0}}\left\|H^{F_{\xi}^{n}}(x, \cdot)\right\|_{\mathcal{C}^{k}\left(U_{s}^{0}, \mathbb{R}^{d_{s}}\right)}$ uniformly in $n$ is essential.

Next, we would like to define the evolution of a foliation $W \in \mathcal{W}_{L}^{r}$ under $T$. Let $W^{n}:=$ $T^{-n} W:=\left\{T^{-n} W_{\alpha}\right\}_{\alpha \in A}$. Clearly, $W^{n} \in \overline{\mathcal{F}}_{\mathcal{C}}^{r}$, but much more is true.

LEMMA 2.9. There exist $n_{0} \in \mathbb{N}$ and $L>0$ such that, for all $n \in \mathbb{N}, n \geq n_{0}, L_{1} \geq L$ and $W \in \mathcal{W}_{L_{1}}^{r}$, we have $W^{n} \in \mathcal{W}_{L_{1} / 2}^{r}$. 
Remark 2.10. By considering an appropriate power of the map, rather than the map itself, we can always reduce to the case $n_{0}=1$. We will do exactly this in the following.

Remark 2.11. From now on, $L$ is fixed so that Lemma 2.9 holds true. Since the choice of $L$ depends only on $T$ and $M$, in the future we will not make the $L$ dependence explicit in the constants.

Lemma 2.9 is proved in Appendix B. In fact, we prove the more general Proposition B.1 which implies Lemma 2.9 (see Remark B.2).

2.5. Test functions. Since we will want to be free to work with high-order derivatives, it is convenient to choose a norm $\|\cdot\|_{\mathcal{C}^{\rho}}, \rho \in \mathbb{N} \cup\{0\}$, equivalent to the standard one, for which $\mathcal{C}^{\rho}$ is a Banach algebra. We thus define the weighted norm in $\mathcal{C}^{\rho}(M, \mathcal{M}(m, n))$, where $\mathcal{M}(m, n)$ is the set of the $m \times n$ (possibly complex valued) matrices,

$$
\begin{aligned}
& \|\varphi\|_{\mathcal{C}^{0}}=\sup _{x \in M} \sup _{i \in\{1, \ldots, n\}} \sum_{j=1}^{m}\left|\varphi_{i, j}(x)\right|, \\
& \|\varphi\|_{\mathcal{C}^{\rho}}=\sum_{k=0}^{\rho} \varpi^{\rho-k} \sup _{|\alpha|=k}\left\|\partial^{\alpha} \varphi\right\|_{\mathcal{C}^{0}},
\end{aligned}
$$

where, $\varpi \geq 2$ is a parameter to be chosen later (see (3.11)), $\alpha$ is a multi-index $\alpha=$ $\left(\alpha_{1}, \cdots, \alpha_{d}\right)$ with $\alpha_{i} \in \mathbb{N} \cup\{0\}$, we denote $|\alpha|=\sum_{i=1}^{d} \alpha_{i}$, and $\partial^{\alpha}=\partial_{x_{1}}^{\alpha_{1}} \cdots \partial_{x_{d}}^{\alpha_{d}}$.

Note that the above definition implies that

$$
\|\varphi\|_{\mathcal{C}^{\rho+1}}=\varpi^{\rho+1}\|\varphi\|_{\mathcal{C}^{0}}+\sup _{i}\left\|\partial_{x_{i}} \varphi\right\|_{\mathcal{C}^{\rho}} .
$$

The next lemma is proved in Appendix A.

Lemma 2.12. For every $\rho, n, m, s \in \mathbb{N}, \psi \in \mathcal{C}^{\rho}(M, \mathcal{M}(m, n))$ and $\varphi \in \mathcal{C}^{\rho}(M, \mathcal{M}(m, s))$,

$$
\|\varphi \psi\|_{\mathcal{C}^{\rho}} \leq\|\varphi\|_{\mathcal{C}^{\rho}}\|\psi\|_{\mathcal{C}^{\rho}}
$$

Moreover, if $\varphi \in \mathcal{C}^{\rho}(M, \mathcal{M}(m, n))$ and $\psi \in \mathcal{C}^{\rho}(M, M)$, then

$$
\|\varphi \circ \psi\|_{\mathcal{C}^{\rho}} \leq \sum_{k=0}^{\rho}\left(\begin{array}{l}
\rho \\
k
\end{array}\right) \varpi^{\rho-k}\|\varphi\|_{\mathcal{C}^{k}} \prod_{i=1}^{k}\left\|(D \psi)^{t}\right\|_{\mathcal{C}^{\rho-i}}
$$

$\left(\mathcal{C}^{\rho}(M, M)\right.$ is defined in the natural manner using the norm (2.7) in the charts $\left(V_{i}, \phi_{i}\right)$. Also, we use the charts to identify $T_{x} M$ with $\mathbb{R}^{d}$. Hence $D \psi \in \mathcal{M}(d, d)$.)

Definition 2.13. For each $\varphi \in \mathcal{C}^{r}\left(M, \mathbb{C}^{l}\right)$ and $W \in \mathcal{F}_{\mathcal{C}}^{r}$, let $\varphi_{\xi, x}(\cdot)=\varphi \circ \phi_{i}^{-1} \circ \mathbb{F}_{\xi}(x, \cdot)$, $q \leq r$, and define (we use the standard notation $\left.\varphi_{\xi, x}=\left(\left(\varphi_{\xi, x}\right)_{1}, \ldots,\left(\varphi_{\xi, x}\right)_{l}\right)\right)$

$$
\|\varphi\|_{q}^{W}:=\sup _{\xi \in M} \sup _{x \in U_{u}^{0}}\|\varphi \xi, x\|_{\mathcal{C}^{q}\left(U_{s}^{0}, \mathbb{C}^{l}\right)}=\sup _{\xi \in M} \sup _{x \in U_{u}^{0}} \sum_{j=1}^{l}\left\|\left(\varphi_{\xi, x}\right)_{j}\right\|_{\mathcal{C}^{q}\left(U_{s}^{0}, \mathbb{C}\right)} .
$$


Remark 2.14. It is easy to verify that a different choice of the charts produces a uniformly equivalent class of norms.

2.6. A class of measures. To be precise, we are going to define a Banach space of distributions. We are interested in measures that belong to such a space. Define

$$
\Omega_{L, q, l}=\left\{(W, \varphi) \in \mathcal{W}_{L}^{r} \times \mathcal{C}^{q}\left(M, \mathbb{C}^{l}\right):\|\varphi\|_{q}^{W} \leq 1\right\}
$$

and lift the dynamics to $\Omega_{L, q, l}$ by $T_{*}(W, \varphi)=\left(T^{-1} W, \varphi \circ T\right)$.

LEMMA 2.15. For each $\sigma \in(\nu, 1)$, there exist constants $A_{0}, B_{0}>0$ such that, for each choice of $\varpi \geq 2$, each $(W, \varphi) \in \Omega_{L, q, l}, q \in\{0, \ldots, r-1\}$ and $n \in \mathbb{N}$,

$$
\begin{aligned}
& \left\|\varphi \circ T^{n}\right\|_{q}^{T^{-n} W} \leq A_{0}\|\varphi\|_{q}^{W}, \\
& \left\|\varphi \circ T^{n}\right\|_{q+1}^{T^{-n} W} \leq A_{0} \sigma^{n q}\|\varphi\|_{q+1}^{W}+B_{0}\|\varphi\|_{q}^{W} .
\end{aligned}
$$

The lemma is proved in Appendix C. Note that Lemmas 2.9 and 2.15 imply that $T_{*} \Omega_{L, q, l} \subset \Omega_{L, q, l}$.

It is now time to define the norms. Given a function $h \in \mathcal{C}^{1}(M, \mathbb{C})$, we define

$$
\begin{aligned}
\|h\|_{0, q} & :=\sup _{(W, \varphi) \in \Omega_{L, q, 1}}\left|\int_{M} h \varphi\right|, \\
\|h\|_{1, q}^{*} & :=\sup _{(W, \varphi) \in \Omega_{L, q+1, d}}\left|\int_{M} h \operatorname{div} \varphi\right|, \\
\|h\|_{1, q}^{-} & :=a\|h\|_{0, q}+\|h\|_{1, q}^{*},
\end{aligned}
$$

for any $q \in \mathbb{N} \cup\{0\}$ and some fixed $a>0$ to be chosen later (see Proposition 3.2).

As already remarked, the differential structure and the volume form are defined via the charts. Thus, to be precise,

$$
\int_{M} h \operatorname{div} \varphi=\sum_{i=1}^{S} \int_{U_{i}} h \circ \phi_{i}^{-1}(z) \vartheta_{i} \circ \phi_{i}^{-1}(z) \sum_{j=1}^{d}\left(\partial_{z_{j}}\left[\varphi_{j} \circ \phi_{i}^{-1}\right]\right)(z) d z .
$$

We are then ready to define the Banach spaces. The space $\mathcal{B}^{0, q}$ is the Banach spaces obtained by completing $\mathcal{C}^{1}(M, \mathbb{R})$ in the $\|\cdot\|_{0, q}$ norm (the completion can be achieved within the space of distributions of order $q$ ). We are not interested in making the same choice for the norm $\|\cdot\|_{1, q}^{-}$since this, in the case when $d_{s}=0$, would yield the Sobolev space $W^{1,1}$ rather than the space of functions of BV that we are interested in. Thus, we use a method analogous to the standard procedure to define BV starting from $W^{1,1}$. First, we define the new norm, for each $h \in \mathcal{B}^{0, q}$,

$$
\|h\|_{1, q}=\lim _{\varepsilon \rightarrow 0} \inf \left\{\|g\|_{1, q}^{-}: g \in \mathcal{C}^{1}(M, \mathbb{R}) \text { and }\|g-h\|_{0, q} \leq \varepsilon\right\} .
$$


We then define $\mathcal{B}^{1, q}:=\left\{h \in \mathcal{B}^{0, q} \mid\|h\|_{1, q}<\infty\right\}$. See $\S 2.7$ of [BKL] for a brief discussion of the general properties of such a construction.

The next lemma explains the sense in which $\mathcal{B}^{1, q}$ is a space of distributions.

LEMMA 2.16. The spaces $\mathcal{B}^{i, q}, i \in\{0,1\}$, are spaces of distributions in the sense that there exist canonical embeddings $\iota_{0, q}: \mathcal{B}^{0, q} \rightarrow\left(\mathcal{C}^{q}\right)^{\prime}$ and $\iota_{1, q}: \mathcal{B}^{1, q} \rightarrow\left(\mathcal{C}^{q+1}\right)^{\prime}$.

Proof. First, note that there exist $C_{q}>0$ such that, for all $\varphi \in \mathcal{C}^{q}$ and $W \in \mathcal{W}_{L}^{r},\|\varphi\|_{q}^{W} \leq$ $C_{q}\|\varphi\|_{\mathcal{C}^{q}}$. In addition, for each $h \in \mathcal{C}^{1}(M, \mathbb{C})$,

$$
\left|\int_{M} h d \omega\right| \leq\|h\|_{0, q} \leq a^{-1}\|h\|_{1, q}
$$

which, by density, implies that $\ell(h)=\int_{M} h d \omega$ belongs to $\left(\mathcal{B}^{0, q}\right)^{\prime} \subset\left(\mathcal{B}^{1, q}\right)^{\prime}$, the duals of $\mathcal{B}^{0, q}$ and $\mathcal{B}^{1, q}$, for each $q \geq 0$. Also, one can easily check that, for each $\varphi \in \mathcal{C}^{q}$ and $h \in \mathcal{B}^{0, q}$, we have $\varphi h \in \mathcal{B}^{0, q}$ and, for each $\varphi \in \mathcal{C}^{q+1}$ and $h \in \mathcal{B}^{1, q}$, we have $\varphi h \in \mathcal{B}^{1, q}$. This implies that $\iota_{0, q}(h)(\varphi):=\ell(\varphi h)$ is well defined for each $\varphi \in \mathcal{C}^{q}$ and $h \in \mathcal{B}^{0, q}$. In addition, for each $h \in \mathcal{C}^{1}(M, \mathbb{C})$,

$$
\left|\iota_{0, q}(h)(\varphi)\right|=\left|\int_{M} \varphi h d \omega\right| \leq\|h\|_{0, q}\|\varphi\|_{q}^{W} \leq C_{q}\|h\|_{0, q}\|\varphi\|_{\mathcal{C}^{q}},
$$

from which, by density, it follows that $\left|\iota_{0, q}(h)(\varphi)\right| \leq C_{q}\|h\|_{0, q}\|\varphi\|_{\mathcal{C}^{q}}$ for all $h \in \mathcal{B}^{0, q}$ and $\varphi \in \mathcal{C}^{q}$ : that is $\iota_{0, q}(h) \in\left(\mathcal{C}^{q}\right)^{\prime}$. Thus $\iota_{0, q}: \mathcal{B}^{0, q} \rightarrow\left(\mathcal{C}^{q}\right)^{\prime}$; it remains to check that it is injective. Suppose that $\iota_{0, q}\left(h_{0}\right)=\iota_{0, q}\left(h_{1}\right)$. Then, for all $\varphi \in \mathcal{C}^{q}$,

$$
\int_{M}\left(h_{0}-h_{1}\right) \varphi=0
$$

which, recalling (2.10), implies that $\left\|h_{0}-h_{1}\right\|_{0, q}=0$.

The other embedding is proved similarly.

Remark 2.17. From now on, we will identify, when needed, the spaces $\mathcal{B}^{i, q}$ with the spaces $\iota_{i, q}\left(\mathcal{B}^{i, q}\right)$ of distributions without further notice. (In general $\iota_{i, q}\left(\mathcal{B}^{i, q}\right)$ is not closed in the $\left(\mathcal{C}^{q+i}\right)^{\prime}$ topology. To see $\iota_{i, q}\left(\mathcal{B}^{i, q}\right)$ as a Banach space we have to induce the norm: if $h \in$ $\iota_{i, q}\left(\mathcal{B}^{i, q}\right)$, then $\|h\|=\left\|\iota_{i, q}^{-1}(h)\right\|_{i, q}$. Obviously, in this way $\iota_{i, q}$ becomes an isomorphism of Banach spaces, hence the possibility to identify them.)

To better understand the $\mathcal{B}^{i, q}$ spaces, it is useful to note that, in special cases, they are simply functions.

Remark 2.18. If $T$ is an expanding map, the $d_{s}=0$, and hence the leaves are just points and $\|\varphi\|_{q}^{W}=|\varphi|_{\infty}$. The reader can easily check that $\mathcal{B}^{0, q}=L^{1}$ and $\mathcal{B}^{1, q}=B V$, as claimed. 
Remark 2.19. By (2.11), it follows that

$$
\begin{aligned}
\sup _{(W, \varphi) \in \Omega_{L, q, 1}}\left|\int_{M} \varphi h\right| & \leq \sup _{|\varphi|_{\infty} \leq 1}\left|\int_{M} h \varphi\right|=\|h\|_{L^{1}}, \\
\sup _{(W, \varphi) \in \Omega_{L, q+1, d}}\left|\int_{M} h \operatorname{div} \varphi\right| & \leq \sup _{\|\varphi\|_{\infty} \leq 1}\left|\int_{M} h \operatorname{div} \varphi\right| \leq\|h\|_{B V} .
\end{aligned}
$$

Thus, by (2.11) and (2.13), $\|h\|_{1, q} \leq C_{a}\|h\|_{B V}$ : that is $L^{1} \subset \mathcal{B}^{0, q}$ and $B V \subset \mathcal{B}^{1, q}$.

Remark 2.20. There is no problem in considering norms with higher smoothness, as in [GL]. We avoid it since it is not relevant for the issue we are presently exploring.

\section{A Lasota-Yorke inequality}

Our first goal is to show that $\mathcal{L}$ is bounded in the $\|\cdot\|_{0, q},\|\cdot\|_{1, q}$ norms, and hence $\mathcal{L}$ extends uniquely to a bounded operator on $\mathcal{B}^{0, q}$ and $\mathcal{B}^{1, q}$.

To prove our basic proposition (a Lasota-Yorke-type inequality), we first need a small approximation lemma.

LEMMA 3.1. There exists $c_{\varpi}>1, \varepsilon_{0}>0$ such that, for each $q \in\{1, \ldots, r\},(W, \varphi) \in$ $\Omega_{L, q, 1}$ and $\varepsilon \in\left(0, \varepsilon_{0}\right)$, there exists $\varphi_{\varepsilon} \in \mathcal{C}^{r}(M, \mathbb{C})$ such that $\left(W, c_{\varpi}^{-1} \varphi_{\varepsilon}\right) \in \Omega_{L, q, 1}$, $\left(W, c_{\varpi}^{-1} \varepsilon \varphi_{\varepsilon}\right) \in \Omega_{L, q+1,1}$ and $\left\|\varphi-\varphi_{\varepsilon}\right\|_{q-1}^{W} \leq C_{q, \varpi} \varepsilon$.

Proof. Let $(W, \varphi) \in \Omega_{L, q, 1}$. Consider a mollifier $\boldsymbol{j}_{\varepsilon}(y)=\varepsilon^{-d_{s}} \boldsymbol{j}\left(\varepsilon^{-1} y\right)$, where $\boldsymbol{j} \in \mathcal{C}^{\infty}$ is supported in a fixed ball. Then, for $\varepsilon \leq \delta_{0} / 2$, and $(x, y) \in U^{0}$, define (see Remark 2.4 for the definition of $\mathbb{F}_{\xi}$ )

$$
\begin{aligned}
\widehat{\varphi}_{i, \varepsilon}(x, y) & =\int_{\mathbb{R}^{d_{s}}} \varphi \circ \phi_{i}^{-1} \circ\left(\mathbb{F}_{\xi}(x, y+z)\right) \boldsymbol{j}_{\varepsilon}(z) d z, \\
\varphi_{\varepsilon} & =\sum_{i} \vartheta_{i} \cdot \widehat{\varphi}_{i, \varepsilon} \circ \mathbb{F}_{\xi}^{-1} \circ \phi_{i} .
\end{aligned}
$$

Clearly, $\varphi_{\varepsilon} \in \mathcal{C}^{r}$, and hence we only have to verify the other two properties. Note that

$$
\varphi_{\varepsilon} \circ \phi_{j}^{-1} \circ \mathbb{F}_{\xi}(x, y)=\sum_{i}\left[\vartheta_{i} \cdot \widehat{\varphi}_{i, \varepsilon} \circ \mathbb{F}_{\xi}^{-1} \circ \phi_{i}\right] \circ \phi_{j}^{-1} \circ \mathbb{F}_{\xi}(x, y) .
$$

By definition, $\mathbb{F}_{\xi}^{-1} \circ \phi_{i} \circ \phi_{j}^{-1} \circ \mathbb{F}_{\xi}(x, y)=\left(h_{i j}(x), g_{i j}(x, y)\right)$ for some $g_{i j}(x, \cdot) \in \mathcal{C}^{r}$. Moreover, $\sup _{i j} \sup _{x}\left\|g_{i j}(x, \cdot)\right\|_{\mathcal{C}^{r}} \leq C$ for some constant $C>0$. Thus

$$
\begin{aligned}
\varphi_{\varepsilon} & \circ \phi_{j}^{-1} \circ \mathbb{F}_{\xi}(x, y) \\
& =\sum_{i} \vartheta_{i} \circ \phi_{j}^{-1} \circ \mathbb{F}_{\xi}(x, y) \int_{\mathbb{R}^{d_{s}}} \varphi \circ \phi_{i}^{-1} \circ \mathbb{F}_{\xi}\left(\left(h_{i j}(x), g_{i j}(x, y)+z\right) \boldsymbol{j}_{\varepsilon}(z) d z .\right.
\end{aligned}
$$

Using the above formula and (3.1), we can estimate

$$
\left\|\varphi_{\varepsilon} \circ \phi_{j}^{-1} \circ \mathbb{F}_{\xi}(x, \cdot)\right\|_{\mathcal{C}^{q}} \leq \sum_{i}\left\|\vartheta_{i} \circ \phi_{j}^{-1} \circ \mathbb{F}_{\xi}(x, \cdot)\right\|_{\mathcal{C}^{r}} \| \widehat{\varphi}_{i, \varepsilon}\left(h_{i j}(x), g_{i j}(x, \cdot) \|_{\mathcal{C}^{q}} \leq c_{\varpi}\right.
$$


for some constant $c_{\varpi}$. On the other hand, recalling (2.7),

$$
\begin{aligned}
& \left\|\varphi_{\varepsilon} \circ \phi_{j}^{-1} \circ \mathbb{F}_{\xi}(x, \cdot)\right\|_{\mathcal{C}^{q+1}} \\
& \leq C_{\varpi}\left\|\varphi_{\varepsilon} \circ \phi_{j}^{-1} \circ \mathbb{F}_{\xi}(x, \cdot)\right\|_{\mathcal{C}^{q}} \\
& \quad+\sum_{i l,|\alpha|=q} C \| \int_{\mathbb{R}^{d_{s}}} \partial_{z_{l}} \partial^{\alpha}\left[\varphi \circ \phi_{i}^{-1} \circ \mathbb{F}_{\xi}\right]\left(\left(h_{i j}(x), z+g_{i j}(x, \cdot)\right)\right. \\
& \quad \times \partial_{y_{l}} g_{i j}(x, \cdot) \prod_{k=1}^{q} \partial_{y_{\alpha_{k}}} g_{i j}(x, \cdot) \cdot j_{\varepsilon}(z) d z \|_{\mathcal{C}^{0}} \\
& \leq C_{\varpi} c_{\varpi}+\sum_{i l,|\alpha|=q} C \| \int_{\mathbb{R}^{d_{s}}} \partial^{\alpha}\left[\varphi \circ \phi_{i}^{-1} \circ \mathbb{F}_{\xi}\right]\left(\left(h_{i j}(x), z+g_{i j}(x, \cdot)\right) \partial_{y_{l}} g_{i j}(x, \cdot)\right. \\
& \quad \times \prod_{k=1}^{q} \partial_{y_{\alpha_{k}}} g_{i j}(x, \cdot) \partial_{z_{j}} j_{\varepsilon}(z) d z \|_{\mathcal{C}^{0}} \\
& \leq C_{\varpi} c_{\varpi}+\|\varphi\|_{q}^{W} C C_{\sharp} \varepsilon^{-1} \leq c_{\varpi} \varepsilon^{-1},
\end{aligned}
$$

provided we chose $c_{\varpi}>2 C C_{\sharp}$ and $\varepsilon_{0}<\left(2 C_{\varpi}\right)^{-1}$. To verify the last inequality, note that there exists a constant $C_{q, w}>0$ such that

$$
\begin{aligned}
& \| \varphi \circ \phi_{i}^{-1} \circ \mathbb{F}_{\xi}\left(\left(h_{i j}(x), z+g_{i j}(x, \cdot)\right)\right. \\
& \quad-\varphi \circ \phi_{i}^{-1} \circ \mathbb{F}_{\xi}\left(\left(h_{i j}(x), g_{i j}(x, \cdot)\right)\left\|_{\mathcal{C}^{q-1}} \leq C_{q, \varpi}\right\| \varphi \|_{q}^{W}|z| .\right.
\end{aligned}
$$

Hence,

$$
\left\|\varphi-\varphi_{\varepsilon}\right\|_{q-1}^{W} \leq C_{q, \varpi} \varepsilon
$$

Proposition 3.2. For each $\theta \in\left(\max \left\{v, \lambda^{-1}\right\}, 1\right)$, we can chose $\varpi>2$ such that there exist constants $a, A, B>0$ such that, for all $h \in \mathcal{C}^{1}(M, \mathbb{C}), q \in\{0, \ldots, r-1\}$,

$$
\left\|\mathcal{L}^{n} h\right\|_{0, q} \leq A\|h\|_{0, q} .
$$

In addition, for all $q \in\{1, \ldots, r-2\}$,

$$
\begin{aligned}
& \left\|\mathcal{L}^{n} h\right\|_{0, q} \leq A \theta^{n}\|h\|_{0, q}+B\|h\|_{0, q+1} ; \\
& \left\|\mathcal{L}^{n} h\right\|_{1, q} \leq A \theta^{n}\|h\|_{1, q}+B\|h\|_{0, q+1} .
\end{aligned}
$$

Proof. Note that if $(W, \varphi) \in \Omega_{L, q, 1}$, then, by (2.7) and (2.2) and for $A$ large enough,

$$
\left|\int_{M} \mathcal{L}^{n} h \varphi\right|=\left|\int_{M} h \varphi \circ T^{n}\right| \leq A\|h\|_{0, q},
$$

from which the first inequality follows. 
For each $\varepsilon>0$ and $(W, \varphi) \in \Omega_{L, q, 1}$, we define $\varphi_{\varepsilon}$ as in Lemma 3.1. Hence,

$$
\begin{aligned}
\left|\int_{M} \mathcal{L}^{n} h \varphi\right| & =\left|\int_{M} h\left(\varphi-\varphi_{\varepsilon}\right) \circ T^{n}\right|+\left|\int_{M} h \varphi_{\varepsilon} \circ T^{n}\right| \\
& \leq\left\|\left(\varphi-\varphi_{\varepsilon}\right) \circ T^{n}\right\|_{q}^{T^{-n} W}\|h\|_{0, q}+\left\|\varphi_{\varepsilon} \circ T^{n}\right\|_{q+1}^{T^{-n} W}\|h\|_{0, q+1} .
\end{aligned}
$$

Then, by Lemmas 2.15 and 3.1,

$$
\begin{aligned}
\left|\int_{M} \mathcal{L}^{n} h \varphi\right| & \leq\left\|\left(\varphi-\varphi_{\varepsilon}\right) \circ T^{n}\right\|_{q}^{T^{-n} W}\|h\|_{0, q}+\left\|\varphi_{\varepsilon} \circ T^{n}\right\|_{q+1}^{T^{-n} W}\|h\|_{0, q+1} \\
& \leq\left(A_{0} \sigma^{q n}\left\|\varphi-\varphi_{\varepsilon}\right\|_{q}^{W}+B_{0}\left\|\varphi-\varphi_{\varepsilon}\right\|_{q-1}^{W}\right)\|h\|_{0, q}+A_{0}\left\|\varphi_{\varepsilon}\right\|_{q+1}^{W}\|h\|_{0, q+1} \\
& \leq\left(2 c_{\varpi}^{-1} A_{0} \sigma^{q n}+C_{q, \varpi} B_{0} \varepsilon\right)\|h\|_{0, q}+A_{0} c_{\varpi} \varepsilon^{-1}\|h\|_{0, q+1} .
\end{aligned}
$$

For each $\theta \in(\sigma, 1)$, there exists $n_{1} \in \mathbb{N}$ and $\varepsilon$ such that $2 A_{0} \sigma^{q n_{1}}+C_{q, \varpi} B_{0} \varepsilon \leq \theta^{2 n_{1}}$. (Recall that, for this statement, we require $q \geq 1$. It is obvious that $q=0$ does not lead to any contraction. This point shows the need to work with a space of distributions rather than a space of measures.) Thus, taking the sup for $(W, \varphi) \in \Omega_{L, q, 1}$, we have, for $n \in\left\{n_{1}, \ldots, 2 n_{1}\right\}$,

$$
\left\|\mathcal{L}^{n} h\right\|_{0, q} \leq \theta^{n}\|h\|_{0, q}+C_{\varpi}\|h\|_{0, q+1} .
$$

Iterating yields that there exists $A_{1}>0$ such that

$$
\begin{aligned}
& \left\|\mathcal{L}^{n} h\right\|_{0, q} \leq \theta^{n}\|h\|_{0, q}+B_{\varpi}\|h\|_{0, q+1} \quad \text { for all } n \geq n_{1}, \\
& \left\|\mathcal{L}^{n} h\right\|_{0, q} \leq A_{1} \theta^{n}\|h\|_{0, q}+B_{\varpi}\|h\|_{0, q+1} \quad \text { for all } n \in \mathbb{N} .
\end{aligned}
$$

Next, we prove the third inequality in the statement of the lemma. For each $(W, \varphi) \in$ $\Omega_{L, q+1, d}$, write

$$
\int_{M} \mathcal{L}^{n} h \operatorname{div} \varphi=\int_{M} h(\operatorname{div} \varphi) \circ T^{n}
$$

Note that, setting $R=\phi_{i} \circ T^{n} \circ \phi_{j}^{-1}$ and recalling equation (2.12),

$$
\operatorname{div}\left(D R^{-1} \circ \phi_{j} \cdot \varphi \circ T^{n}\right)=(\operatorname{div} \varphi) \circ T^{n}+\sum_{l, k=1}^{d} \partial_{l}\left[(D R)^{-1}\right]_{l k} \varphi_{k} \circ \phi_{j} \circ T^{n}
$$

Set $D_{n}=\sup _{l} \sum_{k}\left\|\left[\partial_{l}\left(D R^{-1}\right)_{l, k}\right]\right\|_{\mathcal{C}^{r}}$.

It is then natural to decompose $\varphi$ into an 'unstable' and a 'stable' part. More precisely, consider the 'almost unstable' foliation $\Gamma=\left\{\gamma_{s}\right\}_{s \in \mathbb{R}^{d_{s}}}$ made of the leaves in some chart $\phi_{j}, \gamma_{s}=\{(u, s)\}_{u \in \mathbb{R}^{d_{u}}}$ and its image $T^{n} \Gamma$. The leaves of $T^{n} \Gamma$ can be expressed, in some chart $\phi_{i}$, in the form $\left\{\left(x, \tilde{G}_{n}(x, y)\right\}\right.$ for some function $\tilde{G}_{n}$, smooth in the $x$ variable, with $\left\|\partial_{x} \tilde{G}_{n}\right\| \leq 1$ and the normalization $\tilde{G}_{n}(F(0, y), y)=y$. On the other hand, the leaves of $W$, in the same chart, have the form $\{(F(x, y), y)\}$. It is then natural to consider the change of variables $(x, y)=\Psi_{n}\left(x^{\prime}, y^{\prime}\right)$, where $\left(x, \tilde{G}_{n}\left(x, y^{\prime}\right)\right)=\left(F\left(x^{\prime}, y\right), y\right)$. Writing 
$\varphi=\left(\varphi_{1}, \varphi_{2}\right)$, with $\varphi_{1} \in \mathbb{R}^{d_{u}}, \varphi_{2} \in \mathbb{R}^{d_{s}}$, we consider the decomposition

$$
\begin{aligned}
\varphi \circ \phi_{i}^{-1}(x, y) & =\varphi^{u} \circ \phi_{i}^{-1}(x, y)+\varphi^{s} \circ \phi_{i}^{-1}(x, y) \\
& =\left(v(x, y), \partial_{x} \tilde{G}_{n}\left(x, y^{\prime}\right) v(x, y)\right)+\left(\partial_{y} F\left(x^{\prime}, y\right) w(x, y), w(x, y)\right) .
\end{aligned}
$$

(Since $v$ and $w$ depend on $n$, a more precise notation would be $v_{n}, w_{n}$. We suppress the subscript $n$ to ease notation and since no ambiguity can arise.) That is, setting $\hat{\varphi}=\varphi \circ \phi_{i}^{-1}$,

$$
\begin{aligned}
& v(x, y)=\left(\mathbb{1}-\partial_{y} F\left(x^{\prime}, y\right) \partial_{x} \tilde{G}_{n}\left(x, y^{\prime}\right)\right)^{-1}\left(\hat{\varphi}_{1}(x, y)-\partial_{y} F\left(x^{\prime}, y\right) \hat{\varphi}_{2}(x, y)\right), \\
& w(x, y)=\left(\mathbb{1}-\partial_{x} \tilde{G}_{n}\left(x, y^{\prime}\right) \partial_{y} F\left(x^{\prime}, y\right)\right)^{-1}\left(\hat{\varphi}_{2}(x, y)-\partial_{x} \tilde{G}_{n}\left(x, y^{\prime}\right) \hat{\varphi}_{1}(x, y)\right) .
\end{aligned}
$$

Thus, recalling equation (3.4) and Lemma 2.15,

$$
\begin{aligned}
\left|\int_{M} \mathcal{L}^{n} h \operatorname{div} \varphi\right| \leq & \left|\int_{M} \mathcal{L}^{n} h \operatorname{div} \varphi^{s}\right|+\left|\int_{M} \mathcal{L}^{n} h \operatorname{div} \varphi^{u}\right| \\
\leq & \left|\int_{M} \mathcal{L}^{n} h \operatorname{div} \varphi^{s}\right|+A_{0} D_{n}\|h\|_{0, q+1} \\
& +\left|\int_{M} h \operatorname{div}\left(\left[(D R)^{-1} \circ R^{-1} \circ \phi_{i} \cdot \varphi^{u}\right] \circ T^{n}\right)\right| .
\end{aligned}
$$

To estimate the above terms, our first task is to compute the norm of $\operatorname{div}\left(\varphi^{s}\right),(x, y)=$ $\mathbb{F}\left(x^{\prime}, y\right):=\left(F\left(x^{\prime}, y\right), y\right)$. We start by noticing that

$$
\begin{aligned}
\sum_{i} \partial_{y_{i}}\left[w_{i} \circ \mathbb{F}\right]\left(x^{\prime}, y\right) & =\sum_{i, j}\left(\partial_{x_{j}} w_{i}\right)(x, y) \cdot \partial_{y_{i}} F_{j}\left(x^{\prime}, y\right)+\sum_{i}\left(\partial_{y_{i}} w\right)(x, y) \\
& =\operatorname{div}\left(\varphi^{s}\right)(x, y)-\sum_{i, j, k}\left[\left(\partial_{x_{k}^{\prime}} \partial_{y_{i}} F_{j} \cdot\left(\partial_{x} F\right)_{k j}^{-1}\right) \circ \mathbb{F}^{-1} \cdot w_{i}\right](x, y) .
\end{aligned}
$$

Accordingly, recalling (2.6),

$$
\begin{gathered}
\left.\operatorname{div}\left(\varphi^{s}\right)\left(F\left(x^{\prime}, y\right), y\right)\right)=\left[\sum_{i} \partial_{y_{i}}\left[w_{i} \circ \mathbb{F}\right]+\sum_{i}\left(H_{i}^{F} \cdot w_{i}\right) \circ \mathbb{F}\right]\left(x^{\prime}, y\right), \\
\left.w \circ \mathbb{F}\left(x^{\prime}, y\right)=\left(\mathbb{1}-\partial_{x} \tilde{G}_{n}\left(F\left(x^{\prime}, y\right), y\right) \partial_{y} F\left(x^{\prime}, y\right)\right)^{-1}\left[\varphi_{2} \circ \mathbb{F}-\partial_{x} \tilde{G}_{n} \varphi_{1} \circ \mathbb{F}\right)\right]\left(x^{\prime}, y\right) .
\end{gathered}
$$

Since, $\|w\|_{q+1}^{W} \leq C_{n, \varpi}$, recalling Definition 2.5 for all $|\alpha| \leq q$,

$$
\left|\partial_{y}^{\alpha}\left[\operatorname{div}\left(\varphi^{s}\right) \circ \phi_{i}^{-1} \circ \mathbb{F}\right]\left(x^{\prime}, \cdot\right)\right| \leq C_{n} .
$$

Hence, by (3.2),

$$
\left|\int_{M} \mathcal{L}^{n} h \operatorname{div} \varphi^{s}\right| \leq C_{n}\left\|\mathcal{L}^{n} h\right\|_{0, q} \leq C_{n} A\|h\|_{0, q} .
$$


On the other hand, for each $|\alpha|=q+1$, using (3.5) and (3.6),

$$
\begin{aligned}
& \left|\partial_{y}^{\alpha}\left\{\left[(D R)^{-1} \circ R^{-1} \varphi^{u} \circ \phi_{i}^{-1}\right] \circ \mathbb{F}\right\}\left(x^{\prime}, y\right)\right| \\
& \quad \leq\left|\left(D R \circ R^{-1} \circ \mathbb{F}\left(x^{\prime}, y\right)\right)^{-1}\left(\begin{array}{cc}
\mathbb{1} & 0 \\
0 & \partial_{x} \tilde{G}\left(\mathbb{F}\left(x^{\prime}, y\right)\right)
\end{array}\right) \partial_{y}^{\alpha}\left[v \circ \phi_{i}^{-1} \circ \mathbb{F}\right]\left(x^{\prime}, y\right)\right|+C_{n} \varpi^{-1}\|\varphi\|_{q}^{W},
\end{aligned}
$$

where the last term bounds all the terms with at most $q$ derivatives on $v$. Since the range of the matrix in the line above belongs to the image of the unstable cone under $R$, by (2.2) (and putting in the remainder all the terms with at most $q$ derivatives of $\varphi$ ), we have

$$
\begin{aligned}
& \left|\partial_{y}^{\alpha}\left\{\left[(D R)^{-1} \circ R^{-1} \varphi^{u} \circ \phi_{i}^{-1}\right] \circ \mathbb{F}\right\}\left(x^{\prime}, y\right)\right| \\
& \quad \leq \frac{1+\theta}{c_{0}} \lambda^{-n}\left|\partial_{y}^{\alpha}\left[v \circ \phi_{i}^{-1} \circ \mathbb{F}\right]\left(x^{\prime}, y\right)\right|+C_{n} \varpi^{-1}\|\varphi\|_{q}^{W} \\
& \quad \leq \frac{\left(1+\theta^{2}\right)^{2}}{c_{0}(1-\theta)} \lambda^{-n}\|\varphi\|_{q+1}^{W}+C_{n} \varpi^{-1}\|\varphi\|_{q}^{W} .
\end{aligned}
$$

Accordingly,

$$
\left\|(D R)^{-1} \circ R^{-1} \circ \phi_{i} \cdot \varphi^{u}\right\|_{q+1}^{W} \leq \frac{\left(1+\theta^{2}\right)^{2}}{c_{0}(1-\theta)} \lambda^{-n}\|\varphi\|_{q+1}^{W}+C_{n} \varpi^{-1}\|\varphi\|_{q}^{W} .
$$

Then Lemma 2.15 implies that

$$
\left\|\left[(D R)^{-1} \circ R^{-1} \circ \phi_{i} \cdot \varphi^{u}\right] \circ T^{n}\right\|_{q+1}^{T^{-n} W} \leq \frac{A_{0}\left(1+\theta^{2}\right)^{2}}{c_{0}(1-\theta) \lambda^{n}}\|\varphi\|_{q+1}^{W}+\frac{C_{n}}{\varpi}\|\varphi\|_{q}^{W} .
$$

We can now chose $n_{2} \in \mathbb{N}, n_{2} \geq n_{1}$, such that

$$
\frac{A_{0}(1+\theta)^{2}}{c_{0}(1-\theta)} \lambda^{-n_{2}} \leq \frac{1}{4} \theta^{n_{2}},
$$

and finally we choose $\varpi$ such that

$$
4 \sup _{l \leq 2 n_{2}} C_{l} \theta^{-2 n_{2}} \leq \varpi .
$$

Accordingly, for all $n \in\left\{n_{2}, \ldots, 2 n_{2}\right\}$,

$$
\left\|\left[(D R)^{-1} \circ R^{-1} \circ \phi_{i} \cdot \varphi^{u}\right] \circ T^{n}\right\|_{q+1}^{T^{-n} W} \leq \frac{1}{2} \theta^{n}\|\varphi\|_{q+1}^{W} .
$$

We can then continue the estimate started in (3.7). Recalling (3.9):

$$
\left|\int_{M} \mathcal{L}^{n} h \operatorname{div} \varphi\right| \leq A C_{n}\|h\|_{0, q}+\frac{1}{2} \theta^{n}\|h\|_{1, q}^{*}+A_{0} D_{n_{2}}\|h\|_{0, q+1} .
$$

(Note that, in (3.11), we have chosen $\varpi$ and that the choice depends only on $T$. Thus we can drop the $\varpi$ dependency from all the constants.) Finally, choose $a$ such that $\sup _{l \leq 2 n_{2}} C_{l} A a^{-1} \leq \frac{1}{2} \theta^{2 n_{2}}$. Then, taking the sup on $\varphi, W$, we have, for all $n \in$ $\left\{n_{2}, \ldots, 2 n_{2}\right\}$ and using (3.3),

$$
\left\|\mathcal{L}^{n} h\right\|_{1, q}^{-} \leq \theta^{n}\|h\|_{1, q}^{-}+B_{n_{2}}\|h\|_{0, q+1} .
$$


Then, for each $n \in \mathbb{N}$, we can write $n=k n_{2}+m, m \leq n_{2}$, and iterating the above inequality, we have, for all $n \in \mathbb{N}$,

$$
\left\|\mathcal{L}^{n} h\right\|_{1, q}^{-} \leq A \theta^{n}\|h\|_{1, q}^{-}+B\|h\|_{0, q+1} .
$$

Finally, if $h \in \mathcal{B}^{1, q}$, then there exist $\left\{g_{k}\right\} \in \mathcal{C}^{1}: g_{k} \stackrel{\mathcal{B}^{0, q}}{\rightarrow} h$ and $\left\|g_{k}\right\|_{1, q}^{-} \rightarrow\|h\|_{1, q}$. Since, $\mathcal{L}^{n} g_{k} \in \mathcal{C}^{1}$ and $\mathcal{L}^{n} g_{k} \rightarrow \mathcal{L}^{n} h$ in $\mathcal{B}^{0, q}$,

$$
\begin{aligned}
\left\|\mathcal{L}^{n} h\right\|_{1, q} & \leq \lim _{k \rightarrow \infty}\left\|\mathcal{L}^{n} g_{k}\right\|_{1, q}^{-} \leq A \theta^{n} \lim _{k \rightarrow \infty}\left\|g_{k}\right\|_{1, q}^{-}+B \lim _{k \rightarrow \infty}\left\|g_{k}\right\|_{0, q+1} \\
& =A \theta^{n}\|h\|_{1, q}+B\|h\|_{0, q+1} .
\end{aligned}
$$

This finishes the proof of the second item in the proposition. The proof of the first item of the proposition follows from (3.3) and (3.11).

\section{On the essential spectrum}

In the previous section, we saw that $\mathcal{L}$ (or rather its extension which, with a slight abuse of notation, we still call $\mathcal{L}$ ) belongs both to $L\left(\mathcal{B}^{0, q}, \mathcal{B}^{0, q}\right)$ and $L\left(\mathcal{B}^{1, q}, \mathcal{B}^{1, q}\right)$. Moreover, Proposition 3.2 implies that the spectrum of $\mathcal{L}$ is contained in the unit disk. Next, we want to study the essential spectrum (that is, the complement of the point spectrum with finite multiplicity).

LEMMA 4.1. For $q \in\{1, \ldots, r-2\}$, the essential spectrum of $\mathcal{L}$ on $\mathcal{B}^{1, q}$ is contained in the disk $\left\{z \in \mathbb{C}:|z| \leq \max \left\{\lambda^{-1}, v\right\}\right\}$.

Proof. By Lemmas 2.9 and 2.15, it follows that it suffices to study the sup of $\int_{M} h \varphi$ for $(W, \varphi) \in \Omega_{L / 4, q+1,1}$. Indeed, if $B_{1}^{-}=\left\{h \in \mathcal{B}^{1, q}:\|h\|_{1, q}^{-} \leq 1\right\}$ is relatively compact in the topology associated to the norm $\|h\|_{0, q+1}^{\prime}=\sup _{(W, \varphi) \in \Omega_{L / 4, q+1,1}}\left|\int_{M} h \varphi\right|$, then, by Lemmas 2.9 and 2.15 , there exists $n_{0} \in \mathbb{N}$ such that $\left\|\mathcal{L}^{n_{0}} h\right\|_{0, q+1} \leq\|h\|_{0, q+1}^{\prime}$. Hence, $\mathcal{L}^{n_{0}} B_{1}^{-}$is relatively compact in $\mathcal{B}^{0, q+1}$. Thus $\mathcal{L}^{n_{0}}$ is compact as an operator from $\mathcal{B}^{1, q}$ to $\mathcal{B}^{0, q+1}$ and the lemma follows from Proposition 3.2 and the usual Hennion argument [He] based on Nussbaum's essential spectral formula [Nu] (see [L] for details).

Let us prove the relative compactness of $B_{1}^{-}$. Since we can write

$$
\int_{M} h \varphi=\sum_{i} \int_{M} h \vartheta_{i} \varphi
$$

we can assume, without loss of generality, that $\varphi$ is supported in a given chart $\left(V_{i}, \phi_{i}\right)$. From now on, we will work in such a chart without further mention.

We define $\varphi_{t}$ to be the solution of the heat equation

$$
\begin{aligned}
\partial_{t} \varphi_{t} & =\Delta_{x} \varphi_{t} \quad \text { in } \mathbb{R}^{d} \times[0,1], \\
\varphi_{0} & =\varphi .
\end{aligned}
$$

That is,

$$
\varphi_{t}(x, y)=\frac{1}{(4 \pi t)^{d_{u} / 2}} \int_{\mathbb{R}^{d_{u}}} e^{-\left(|\zeta|^{2}\right) / 4 t} \varphi(x-\zeta, y) d \zeta
$$


Then, for each small $\varepsilon>0$,

$$
\begin{aligned}
\int_{M} h \varphi & =\int_{M} h \varphi_{\varepsilon}-\int_{0}^{\varepsilon} d t \int_{M} h \partial_{t} \varphi_{t}=\int_{V_{i}} h \varphi_{\varepsilon}-\int_{0}^{\varepsilon} d t \int_{V_{i}} h \operatorname{div} \nabla_{x} \varphi_{t}(x, y) \\
& =\int_{V_{i}} h \varphi_{\varepsilon}+\int_{0}^{\varepsilon} d t \frac{1}{(4 \pi t)^{d_{u} / 2}} \int_{V_{i}} d x d y \int_{\mathbb{R}^{d_{u}}} d \zeta e^{-\left(|\zeta|^{2} / 4 t\right)} h \operatorname{div} \nabla_{\zeta} \varphi(x-\zeta, y) \\
& =\int_{V_{i}} h \varphi_{\varepsilon}+\int_{0}^{\varepsilon} d t \frac{1}{(4 \pi t)^{d_{u} / 2}} \int_{\mathbb{R}^{d_{u}}} d \zeta e^{-\left(|\zeta|^{2}\right) / 4 t} \int_{V_{i}} h \operatorname{div} \frac{\zeta}{2 t} \varphi^{\zeta},
\end{aligned}
$$

where, in the last line, $\varphi^{\zeta}(x, y):=\varphi(x-\zeta, y)$ and we have integrated by parts with respect to $\zeta$. Next, for each $\zeta \in \mathbb{R}^{d_{u}}$, we define the foliation $\mathbb{F}_{\zeta}(x, y):=(F(x-\zeta, y)+$ $\zeta, y)$. Note that the foliation $W^{\zeta}$ defined by $\mathbb{F}_{\zeta}$ belongs to $\mathcal{W}_{L / 4}^{r}$. Then $\varphi^{\zeta} \circ \mathbb{F}_{\zeta}(x, y)=$ $\varphi(F(x-\zeta, y), y)$, which implies that $\left\|\varphi^{\zeta}\right\|_{q+1}^{W_{\zeta}} \leq 1$. Hence,

$$
\int_{M} h \varphi=\int_{V_{i}} h \varphi_{\varepsilon}+\mathcal{O}\left(\|h\|_{1, q}^{-} \varepsilon\right) .
$$

In addition, by (4.1) and integrating $r$ times by parts,

$$
\left|\varphi_{\varepsilon}(\cdot, y)\right|_{\mathcal{C}^{r}} \leq \frac{C_{r}}{(4 \pi \varepsilon)^{d_{u} / 2}} \int_{\mathbb{R}^{d_{u}}} e^{-\left(|\zeta|^{2}\right) / 4 \varepsilon}\left(\varepsilon^{-(r / 2)}+\varepsilon^{-r}\|\xi\|^{r}\right)\|\varphi\|_{\mathcal{C}^{0}} d \zeta \leq C \varepsilon^{-(r / 2)} .
$$

Moreover, recalling (4.1), Definition 2.5 and Lemma B.7,

$$
\begin{aligned}
\varphi_{\varepsilon} \circ \mathbb{F}(x, y) & =\frac{1}{(4 \pi \varepsilon)^{d / 2}} \int_{\mathbb{R}^{d_{u}}} e^{-\left(|F(x, y)-\zeta|^{2}\right) / 4 \varepsilon} \varphi(\zeta, y) d \zeta \\
& =\frac{1}{(4 \pi \varepsilon)^{d / 2}} \int_{\mathbb{R}^{d_{u}}} e^{-\left(|F(x, y)-F(\xi, y)|^{2}\right) / 4 \varepsilon} \varphi(F(\xi, y), y) \operatorname{det}\left(\partial_{x} F\right)(\xi, y) d \xi,
\end{aligned}
$$

which readily implies that $\left\|\varphi_{\varepsilon}\right\|_{r}^{W} \leq C \varepsilon^{-r}$. This, by [J], implies that $\left|\varphi_{\varepsilon}\right| \mathcal{C}^{r} \leq C \varepsilon^{-r}$. Thus, recalling (4.2), we have, for each $\varepsilon>0$,

$$
\|h\|_{0, q+1} \leq C \varepsilon^{-r}\|h\|_{\left(\mathcal{C}^{r}\right)^{\prime}}+C\|h\|_{1, q}^{-} \varepsilon .
$$

Since $\left(\mathcal{C}^{q+1}\right)^{\prime}$ embeds compactly in $\left(\mathcal{C}^{r}\right)^{\prime}$ and Lemma 2.16 implies that $B_{1}^{-}$is a bounded subset of $\left(\mathcal{C}^{q+1}\right)^{\prime}$, it follows that $B_{1}^{-}$is relatively compact in $\left(\mathcal{C}^{r}\right)^{\prime}$. From this and equation (4.3), the relative compactness of $B_{1}^{-}$in $\mathcal{B}^{0, q+1}$ readily follows. Hence the lemma follows.

\section{On the peripheral spectrum}

The previous section implies that, for each $\beta \in\left(\max \left\{\lambda^{-1}, \nu\right\}, 1\right)$, the spectral decomposition is

$$
\mathcal{L}=\sum_{j=1}^{L_{\beta}} \lambda_{j} \Pi_{j}+R
$$

where $\Pi_{j} \Pi_{k}=\delta_{j k} \Pi_{k}^{2}, \Pi_{j} R=R \Pi_{j}=0$, each $\Pi_{j}$ is a finite rank operator, and the spectral radius of $R$ is bounded by $\beta$. 
LeMma 5.1. One is an eigenvalue of $\mathcal{L}$. Letting $h_{*}:=\Pi_{1} 1, h_{*}$ is a measure. In addition, the peripheral spectrum of $\mathcal{L}$ consists of finitely many finite groups.

Proof. Since $\ell$ is an eigenvalue of the dual of $\mathcal{L}$ (the Lebesgue measure being the eigenvector), it must belong to the spectrum of $\mathcal{L}$. Next, we choose $\beta$ in representation (5.1) to be large enough so that, for all the eigenvectors, we have $\left|\lambda_{j}\right|=1$. In this case, since the operator is power bounded, the $\Pi_{j}$ cannot contain Jordan blocks. Thus $\Pi_{j} \Pi_{k}=\delta_{j k} \Pi_{k}$. A simple computation based on (5.1) shows that

$$
\lim _{n \rightarrow \infty} \frac{1}{n} \sum_{k=0}^{n-1} e^{-i \vartheta} \mathcal{L}^{k}= \begin{cases}0 & \text { if } e^{i \vartheta} \notin \sigma(\mathcal{L}) \\ \Pi_{j} & \text { if } e^{i \vartheta}=\lambda_{j}=: e^{i \vartheta_{j}}\end{cases}
$$

For each $\varphi \in \mathcal{C}^{q}$,

$$
\left|\int_{M} h_{*} \varphi\right| \leq \lim _{n \rightarrow \infty} \frac{1}{n} \sum_{k=0}^{n-1} \int_{M} \mathcal{L}^{k} 1|\varphi|=\lim _{n \rightarrow \infty} \frac{1}{n} \sum_{k=0}^{n-1} \int_{M}\left|\varphi \circ T^{k}\right| \leq|\varphi|_{\infty} .
$$

In other words, $h_{*}$ defines a measure. Then let $h \in \mathcal{C}^{1}$ and $\varphi \in \mathcal{C}^{q}, \varphi \geq 0$.

$$
\begin{aligned}
\left|\int_{M} \Pi_{j} h \varphi\right| & \leq \lim _{n \rightarrow \infty} \frac{1}{n} \sum_{k=0}^{n-1} \int_{M} \mathcal{L}^{k}|h| \varphi=\lim _{n \rightarrow \infty} \frac{1}{n} \sum_{k=0}^{n-1} \int_{M}|h| \varphi \circ T^{k} \\
& \leq|h|_{\infty} \lim _{n \rightarrow \infty} \frac{1}{n} \sum_{k=0}^{n-1} \int_{M} \mathcal{L}^{k} 1 \varphi=|h|_{\infty} \int_{M} h_{*} \varphi .
\end{aligned}
$$

Moreover, by a similar computation,

$$
\left|\int_{M} \Pi_{j} h \varphi\right| \leq \lim _{n \rightarrow \infty} \frac{1}{n} \sum_{k=0}^{n-1} \int_{M} \mathcal{L}^{k}|h| \varphi \leq|\varphi|_{\infty} \int_{M}|h| .
$$

This implies that $\Pi_{j} h=\sum_{l=1}^{n_{j}} \psi_{j, l} h_{*} \int_{M} h \phi_{j, l}$, where $\psi_{j, l}, \phi_{j, l} \in L^{\infty}(M)$. Note that $\Pi_{k} \Pi_{m}=\delta_{k m} \Pi_{k}$ implies that

$$
\int_{M} \phi_{k, l} \psi_{m, l^{\prime}} h_{*}=\delta_{k, m} \delta_{l, l^{\prime}}
$$

Accordingly, for all $g, h \in \mathcal{C}^{r}$,

$$
\begin{aligned}
\sum_{l=1}^{n_{j}} \int_{M} g \psi_{j, l} h_{*} \int_{M} \phi_{j, l} \circ T h & =\int_{M} g \Pi_{j} \mathcal{L} h=e^{i \vartheta_{j}} \int_{M} g \Pi_{j} h \\
& =e^{i \vartheta_{j}} \sum_{l=1}^{n_{j}} \int_{M} g \psi_{j, l} h_{*} \int_{M} h \phi_{j, l} .
\end{aligned}
$$


It follows that $\phi_{j, l} \circ T=e^{i \vartheta_{j}} \phi_{j, l}, \omega$ almost surely. On the other hand,

$$
\begin{aligned}
\sum_{l=1}^{n_{j}} \int_{M} g \mathcal{L} \psi_{j, l} h_{*} \int_{M} \phi_{j, l} h & =\int_{M} g \mathcal{L} \Pi_{j} h=e^{i \vartheta_{j}} \int_{M} g \Pi_{j} h \\
& =e^{i \vartheta_{j}} \sum_{l=1}^{n_{j}} \int_{M} g \psi_{j, l} h_{*} \int_{M} h \phi_{j, l} .
\end{aligned}
$$

By the arbitrariness of $g, h$, it follows that

$$
e^{i \vartheta_{j}} \psi_{j, l} h_{*}=\mathcal{L} \psi_{j, l} h_{*}=\psi_{j, l} \circ T^{-1} \mathcal{L} h_{*}=\psi_{j, l} \circ T^{-1} h_{*},
$$

which implies that $\psi_{j, l} \circ T^{-1}=e^{i \vartheta_{j}} \psi_{j, l}, h_{*} d \omega$ almost surely. Note that this implies that, for all $k \in \mathbb{N}, \psi_{j, l}^{k} \circ T^{-1}=e^{i \vartheta_{j} k} \psi_{j, l}^{k}$. Thus $\mathcal{L}\left(\psi_{j, l}^{k} h_{*}\right)=\psi_{j, l}^{k} \circ T^{-1} \mathcal{L} h_{*}=e^{i \vartheta_{j} k} \psi_{j, l}^{k} h_{*}$. By an approximation argument, one can prove that $\psi_{j, l}^{k} h_{*} \in \mathcal{B}^{1, q}$. But then it follows that $\left\{e^{i \vartheta_{j} k}\right\} \subset \sigma(\mathcal{L})$ and since the operator is quasi-compact it can have only finitely many isolated eigenvalues. Thus, we must have $\vartheta_{j}=2 \pi k_{j} / n_{j}$, which concludes the proof.

Lemma 5.1 implies that there exists $\bar{m} \in \mathbb{N}$ such that the peripheral spectrum of $\mathcal{L}^{\bar{m}}$ consists of only the eigenvalue 1 with associated eigenprojector $\bar{\Pi}=\sum_{l=1}^{N} \psi_{l} h_{*} \int_{M} h \phi_{l}$, where $\psi_{l} \in\left\{\psi_{j, i}\right\}$ and $\phi_{l} \in\left\{\phi_{j, i}\right\}$. Moreover, (5.3) implies that

$$
\int_{M} \phi_{l} \psi_{l^{\prime}} h_{*}=\delta_{l, l^{\prime}} .
$$

Accordingly, the rest of the spectrum will be contained in a disk strictly smaller than one: that is, $\mathcal{L}^{\bar{m}}=\bar{\Pi}+Q$, where $\left\|Q^{n}\right\|_{1, q} \leq C \sigma^{n}$ for some $C>0$ and $\sigma \in(0,1)$. In addition, note that (5.2) implies that $\bar{\Pi} 1=h_{*}$.

A more precise result can be easily obtained.

LEMMA 5.2. The ergodic decomposition of $h_{*}$ corresponds to the spectral decomposition for the Anosov map and consists of the physical measures.

Proof. Let $\bar{\Pi}^{\prime} h=\sum_{l=1}^{N} \phi_{l} \int_{M} \psi_{l} h_{*} h$ and recall that $\phi_{l} \circ T=\phi_{l}$. For each $h \in$ $\mathcal{C}^{q}(M, \mathbb{R})$, let $\hat{h}=h-\bar{\Pi}^{\prime} h$. Then

$$
\begin{aligned}
\int_{M}\left|\frac{1}{n} \sum_{k=0}^{n-1} h \circ T^{\bar{m} k}-\bar{\Pi}^{\prime} h\right|^{2}=\int_{M}\left|\frac{1}{n} \sum_{k=0}^{n-1} \hat{h} \circ T^{\bar{m} k}\right|^{2} \\
=\sum_{k, j=0}^{n-1} \frac{1}{\bar{n}^{2}} \int_{M} \hat{h} \circ T^{\bar{m} k} \hat{h} \circ T^{\bar{m} j} \\
=\sum_{k=0}^{n-1} \frac{1}{n^{2}} \int_{M} \mathcal{L}^{\bar{m} k} 1 \hat{h}^{2}+2 \sum_{k>j=0}^{n-1} \frac{1}{n^{2}} \int_{M} \hat{h} \mathcal{L}^{\bar{m} k-\bar{m} j} \hat{h} \mathcal{L}^{\bar{m} j} 1
\end{aligned}
$$




$$
\begin{aligned}
& =\mathcal{O}\left(\frac{1}{n}\right)+2 \sum_{j=0}^{n-1} \sum_{l=1}^{n-j-1} \frac{1}{n^{2}} \int_{M} \hat{h} \mathcal{L}^{\bar{m} l} \hat{h} \mathcal{L}^{\bar{m} j} 1 \\
& =\mathcal{O}\left(\frac{1}{n}\right)+2 \sum_{j=0}^{n-1} \sum_{l=1}^{n-j-1} \frac{1}{n^{2}} \int_{M} \hat{h} \bar{\Pi} \hat{h} \bar{\Pi} 1+C \sum_{j=0}^{n-1} \sum_{l=1}^{n-j-1} \frac{1}{n^{2}}\left(\sigma^{l}+\sigma^{j}\right) \\
& =\mathcal{O}\left(\frac{1}{n}\right)+2 \sum_{j=0}^{n-1} \sum_{l=1}^{n-j-1} \frac{1}{n^{2}} \int_{M} \hat{h} \bar{\Pi} \hat{h} \bar{\Pi} 1 .
\end{aligned}
$$

Next, note that, recalling (5.4),

$$
\begin{aligned}
\int_{M} \hat{h} \bar{\Pi} \hat{h} \bar{\Pi} 1 & =\int_{M} \hat{h} \bar{\Pi} \hat{h} h_{*} \\
& =\sum_{l=1}^{N} \int_{M} h \psi_{l} h_{*} \int_{M} \phi_{l} \hat{h} h_{*}-\sum_{l=1}^{N} \sum_{j=1}^{N} \int_{M} \psi_{l} h_{*} h \int_{M} \phi_{l} \psi_{j} h_{*} \int_{M} \phi_{j} \hat{h} h_{*}=0 .
\end{aligned}
$$

It follows that

$$
\int_{M}\left|\frac{1}{n} \sum_{k=0}^{n-1} h \circ T^{\bar{m} k}-\bar{\Pi}^{\prime} h\right|^{2} \leq C_{h} n^{-1} .
$$

By Chebyshev, this implies that

$$
\omega\left(\left\{x \in M:\left|\frac{1}{n} \sum_{k=0}^{n-1} h \circ T^{\bar{m} k}-\bar{\Pi}^{\prime} h\right| \geq \varepsilon\right\}\right) \leq \frac{C_{h}}{\varepsilon^{2} n} .
$$

Thus, if we consider $\alpha \in(0,1)$ and the set $I=\cup_{k \in \mathbb{N}}\left\{2^{k}+j 2^{\alpha k}\right\}_{0 \leq j<2^{(1-\alpha) k}}$, each sequence $\left\{n_{j}\right\} \subset I$ will have limit $\omega$ almost surely by a standard Borel-Cantelli argument. On the other hand, since $h$ is bounded, this readily implies that

$$
\lim _{n \rightarrow \infty} \frac{1}{n} \sum_{k=0}^{n-1} h \circ T^{\bar{m} k}=\bar{\Pi}^{\prime} h=\sum_{l=1}^{N} \phi_{l} \int_{M} \psi_{l} h_{*} h \quad \omega \text {-almost surely. }
$$

By an obvious approximation argument, the same can be proved for each $h \in \mathcal{C}^{0}(M, \mathbb{R})$. This implies that the ergodic decomposition of $h_{*}$ consists of the physical measures. It is well known that these are the SRB measures of the system.

Remark 5.3. If the map is topologically transitive, then the physical measure is unique and so are the physical measures of the powers of the map. Hence the map is mixing, and no other eigenvalue of modulus one exists. Thus, the transfer operator has a spectral gap and the map is exponentially mixing for BV observables.

Acknowledgement. WB and CL would like to thank the Heilbronn Institute for Mathematical Research where part of this work took place. WB would also like to thank the Department of Mathematics, University of Rome Tor Vergata, where some of this work was carried out, for its hospitality. CL would like to thank Viviane Baladi, Sebastien Gouëzel and Mark Demers for helpful discussions. CL acknowledges the MIUR Excellence 
Department Project awarded to the Department of Mathematics, University of Rome Tor Vergata, CUP E83C18000100006. The paper was partially supported by the Grant PRIN 2017S35EHN.

\section{A. Appendix. Norms estimates}

We provide a few tools on how to estimate $\mathcal{C}^{q}$ norms of products and compositions of functions. These are well-known facts, yet it is not easy to find in the literature the exact statements needed here, so we provide them for the reader's convenience.

Proof of Lemma 2.12. Let $\varphi, \psi \in \mathcal{C}^{\rho}(M, \mathbb{C})$. First, we prove, by induction on $\rho$, that

$$
\sup _{|\alpha|=\rho}\left\|\partial^{\alpha}(\varphi \psi)\right\|_{\mathcal{C}^{0}} \leq \sum_{k=0}^{\rho}\left(\begin{array}{l}
\rho \\
k
\end{array}\right) \sup _{|\beta|=\rho-k}\left\|\partial^{\beta} \varphi\right\|_{\mathcal{C}^{0}} \sup _{|\gamma|=k}\left\|\partial^{\gamma} \psi\right\|_{\mathcal{C}^{0}}
$$

Indeed, it is trivial for $\rho=0$ and

$$
\begin{aligned}
\left\|\partial_{x_{i}} \partial^{\alpha}(\varphi \psi)\right\|_{\mathcal{C}^{0}}= & \left\|\partial^{\alpha}\left(\psi \partial_{x_{i}} \varphi+\varphi \partial_{x_{i}} \psi\right)\right\|_{\mathcal{C}^{0}} \\
\leq & \sum_{k=0}^{\rho}\left(\begin{array}{l}
\rho \\
k
\end{array}\right) \sup _{|\beta|=\rho-k}\left\|\partial^{\beta} \partial_{x_{i}} \varphi\right\|_{\mathcal{C}^{0}} \sup _{|\gamma|=k}\left\|\partial^{\gamma} \psi\right\|_{\mathcal{C}^{0}} \\
& +\sum_{k=0}^{\rho}\left(\begin{array}{l}
\rho \\
k
\end{array}\right) \sup _{|\beta|=\rho-k}\left\|\partial^{\beta} \partial_{x_{i}} \psi\right\|_{\mathcal{C}^{0}} \sup _{|\gamma|=k}\left\|\partial^{\gamma} \varphi\right\|_{\mathcal{C}^{0}} \\
\leq & \sum_{k=0}^{\rho}\left(\begin{array}{l}
\rho \\
k
\end{array}\right) \sup _{|\beta|=\rho-k+1}\left\|\partial^{\beta} \varphi\right\|_{\mathcal{C}^{0}} \sup _{|\gamma|=k}\left\|\partial^{\gamma} \psi\right\|_{\mathcal{C}^{0}} \\
& +\sum_{k=1}^{\rho+1}\left(\begin{array}{c}
\rho \\
\rho+1-k
\end{array}\right) \sup _{|\gamma|=k}\left\|\partial^{\gamma} \psi\right\|_{\mathcal{C}^{0}} \sup _{|\beta|=\rho-k+1}\left\|\partial^{\beta} \varphi\right\|_{\mathcal{C}^{0}},
\end{aligned}
$$

from which (A.1) follows by taking the sup on $\alpha, i$ and since $\left(\begin{array}{c}\rho \\ k\end{array}\right)+\left(\begin{array}{c}\rho \\ \rho+1-k\end{array}\right)=\left(\begin{array}{c}\rho+1 \\ k\end{array}\right)$. The first statement of the lemma readily follows: that is,

$$
\begin{aligned}
\|\varphi \psi\|_{\mathcal{C}^{\rho}} & =\sum_{k=0}^{\rho} \varpi^{\rho-k} \sum_{j=0}^{k}\left(\begin{array}{l}
k \\
j
\end{array}\right) \sup _{|\beta|=k-j}\left\|\partial^{\beta} \varphi\right\|_{\mathcal{C}^{0}} \sup _{|\gamma|=j}\left\|\partial^{\gamma} \psi\right\|_{\mathcal{C}^{0}} \\
& =\sum_{j=0}^{\rho} \sum_{k=j}^{\rho} \varpi^{\rho-k}\left(\begin{array}{l}
k \\
j
\end{array}\right) \sup _{|\beta|=k-j}\left\|\partial^{\beta} \varphi\right\|_{\mathcal{C}^{0}} \sup _{|\gamma|=j}\left\|\partial^{\gamma} \psi\right\|_{\mathcal{C}^{0}} \\
& \leq \sum_{j=0}^{\rho} \sum_{l=0}^{\rho-j}\left(\begin{array}{c}
j+l \\
j
\end{array}\right) \varpi^{\rho-j-l} \sup _{|\beta|=l}\left\|\partial^{\beta} \varphi\right\|_{\mathcal{C}^{0}} \sup _{|\gamma|=j}\left\|\partial^{\gamma} \psi\right\|_{\mathcal{C}^{0}} \leq\|\varphi\|_{\mathcal{C}^{\rho}\|\psi\|_{\mathcal{C}^{\rho}}}
\end{aligned}
$$

since $\left(\begin{array}{c}j+l \\ j\end{array}\right) \leq 2^{j+l} \leq \varpi^{\rho}$. The extension to functions with values in the matrices is trivial since we have chosen a norm in which the matrices form a normed algebra.

To prove the second inequality of the lemma, we proceed again by induction on $\rho$. The case $\rho=1$ is trivial from the definition of the norm. We assume that the statement is true 
for every $k \leq \rho$ and show it for $\rho+1$. By the definition of $\|\cdot\|_{\mathcal{C}^{\rho}}$,

$$
\|\varphi \circ \psi\|_{\mathcal{C}^{\rho+1}} \leq \varpi^{\rho+1}\|\varphi\|_{\mathcal{C}^{0}}+\sup _{i}\left\|\partial_{x_{i}}(\varphi \circ \psi)\right\|_{\mathcal{C}^{\rho}} .
$$

By hypothesis (below, we use the elementary fact that $\left.\left(\begin{array}{c}\rho \\ q-1\end{array}\right) \leq\left(\begin{array}{c}\rho+1 \\ q\end{array}\right), q \leq \rho+1\right)$,

$$
\begin{aligned}
\left\|\partial_{x_{i}}(\varphi \circ \psi)\right\|_{\mathcal{C}^{\rho}} & \leq \sup _{j}\left\|\left(\partial_{x_{j}} \varphi\right) \circ \psi\right\|_{\mathcal{C}^{\rho}}\left\|(D \psi)^{t}\right\|_{\mathcal{C}^{\rho}} \\
& \leq \sum_{k=0}^{\rho}\left(\begin{array}{c}
\rho \\
k
\end{array}\right) \varpi^{\rho-k}\|\varphi\|_{\mathcal{C}^{k+1}} \prod_{i=0}^{k}\left\|(D \psi)^{t}\right\|_{\mathcal{C}^{\rho-i}} \\
& \leq \sum_{q=1}^{\rho+1}\left(\begin{array}{c}
\rho+1 \\
q
\end{array}\right) \varpi^{\rho+1-q}\|\varphi\|_{\mathcal{C}^{q}} \prod_{j=1}^{q}\left\|(D \psi)^{t}\right\|_{\mathcal{C}^{\rho+1-j}} .
\end{aligned}
$$

Finally, notice that the term with $q=0$ in the sum above is exactly the first term of the right-hand side of (A.2), which gives the result for $\rho+1$ and proves the induction.

Remark A.1. Notice that, for $\varphi, \psi \in \mathcal{C}^{\rho}$, the definition of the norm and Lemma 2.12 imply that

$$
\|\varphi \circ \psi\|_{\mathcal{C}^{\rho}} \leq\|\varphi\|_{\mathcal{C}^{\rho}} \sum_{j=0}^{\rho}\left(\begin{array}{l}
\rho \\
j
\end{array}\right) \varpi^{\rho-j}\left\|(D \psi)^{t}\right\|_{\mathcal{C}^{\rho-1}}^{j}
$$

\section{B. Appendix. Foliations: regularity properties}

This appendix is devoted to proving Lemma 2.9 and a few other technical lemmas. In essence, we study the behaviour of foliations under iteration. This is very similar to what is done in the construction of the invariant foliations and in the study of their regularity properties, including the regularity of the holonomies. The reason to redo it here without appealing to the literature is that we need these facts in an unconventional form. In particular, we could not find anywhere in the literature the infinitesimal characterization of the holonomy used here, which is a characterization, hopefully, very helpful in the study of discontinuous hyperbolic maps.

Given such a new twist in the theory, we think it is appropriate to present a more general result: we will control also the regularity of the leaves, and of their tangent spaces, in the unstable direction, although this is not needed in the present paper. More precisely, we will see that the derivatives of the foliation along the leaves vary in a $\tau_{0}$-Hölder manner. The optimal $\tau_{0}$ is well known to depend on a bunching condition [PSW, HW]. We ignore this issue since it largely exceeds our present purposes and to investigate it would entail a lengthier argument. Note that Lemma 2.9 is a special case of Proposition B.1 below when choosing $\tau=0$.

Let $\tau \in(0,1)$. Given $\varphi: M \rightarrow \mathbb{R}$, we define, for some $\delta_{\star}>0$,

$$
\|\varphi\|_{\mathcal{C}^{\tau}}=\|\varphi\|_{\mathcal{C}^{0}}+\sup _{\xi \in M} \sup _{\substack{\left(\xi, \xi^{\prime}\right) \leq \delta_{\star} \\ \xi \neq \xi^{\prime}}} \frac{\left|\varphi(\xi)-\varphi\left(\xi^{\prime}\right)\right|}{d\left(\xi, \xi^{\prime}\right)^{\tau}},
$$


where $d(\cdot, \cdot)$ is the Riemannian distance and $\delta_{\star} \in(0,1)$. Also, for each $r \in \mathbb{R}, r=q+\tau$ and $q \in \mathbb{N} \cup\{0\}$, we define

$$
\|\varphi\|_{r}=\sum_{k=0}^{q} \sup _{|\alpha|=k}\left\|\partial^{\alpha} \varphi\right\|_{\mathcal{C}^{0}}+\sup _{|\alpha|=q}\left\|\partial^{\alpha} \varphi\right\|_{\mathcal{C}^{\tau}} .
$$

Note that, for $\tau=0$, the above corresponds to (2.7) with the choice $\varpi=1$. (This choice of a different equivalent norm, limited to this appendix, is slightly annoying, but convenient.)

Note that $\|\varphi \cdot \phi\|_{\mathcal{C}^{\tau}} \leq\|\varphi\|_{\mathcal{C}^{\tau}}\|\phi\|_{\mathcal{C}^{\tau}}$, so $\mathcal{C}^{\tau}$ is a Banach algebra. The same holds for matrix valued functions.

Although the above norms are all equivalent, they depend on $\delta_{\star}$. We will choose $\delta_{\star}$ in (B.11). Let $T \in \mathcal{C}^{r}$ and define, for $\tau \in[0,1)$,

$$
\begin{aligned}
\mathcal{W}_{L}^{r, \tau}:=\{ & \left\{W \in \overline{\mathcal{F}}_{\mathcal{C}}^{r}: \sup _{\xi ; y \in U_{s}^{0}}\left\|\partial_{y}^{\alpha} F_{\xi}(\cdot, y)\right\|_{\mathcal{C}^{0}\left(U_{u}^{0}, \mathbb{R}^{d_{u}}\right)} \leq L^{(|\alpha|-1)^{2}}, 2 \leq|\alpha| \leq r ;\right. \\
& \sup _{\xi} \sup _{y \in U_{s}^{0}}\left\|\partial_{y}^{\alpha} F_{\xi}(\cdot, y)\right\|_{\mathcal{C}^{\tau}\left(U_{u}^{0}, \mathbb{R}^{d_{u}}\right)} \leq 2 L^{|\alpha|^{2}},|\alpha| \leq r-1 ; \\
& \sup _{\xi} \sup _{y \in U_{s}^{0}}\left\|\partial_{y}^{\alpha} H^{F_{\xi}}(\cdot, y)\right\|_{\mathcal{C}^{0}\left(U_{u}^{0}, \mathbb{R}^{d_{s}}\right)} \leq L^{(|\alpha|+1)^{2}},|\alpha| \leq r-2 ; \\
& \left.\sup _{\xi} \sup _{y \in U_{s}^{0}}\left\|\partial_{y}^{\alpha} H^{F_{\xi}}(\cdot, y)\right\|_{\mathcal{C}^{\tau}\left(U_{u}^{0}, \mathbb{R}^{d_{s}}\right)} \leq 2 L^{(|\alpha|+2)^{2}},|\alpha| \leq r-3\right\} .
\end{aligned}
$$

Note that, recalling the cone definition (2.3), Definition 2.3 and the subsequent definition of $F$, it follows that, for $W \in \mathcal{W}_{L}^{r, \tau}$, the corresponding $F$ must satisfy $\left\|\partial_{y} F\right\| \leq 1$ and $\|F(x, y)\| \leq\|x\|+\|y\|$.

Proposition B.1. There exist $\tau_{0} \in(0,1), \delta_{\star}>0, n_{0} \in \mathbb{N}$ and $L>0$ such that, for all $n \in \mathbb{N}, n \geq n_{0}, L_{1} \geq L$ and $W \in \mathcal{W}_{L_{1}}^{r, \tau_{0}}$, we have $W^{n} \in \mathcal{W}_{L_{1} / 2}^{r, \tau_{0}}$.

Remark B.2. Note that, for $\tau=0$, the conditions in $\mathcal{W}_{L}^{r, \tau}$ reduce to a control on the sup norm of the derivatives $\partial_{y}^{\alpha} F(\cdot, y)$ exactly as in the definition of $\mathcal{W}_{L}^{r}$ in Definition 2.5. The control stated in Proposition B.1 on $\partial_{y}^{\alpha} F(\cdot, y)$ is known. As for $\partial_{y}^{\alpha} H^{F}(\cdot, y)$, we are not aware of this result anywhere in the literature.

Remark B.3. Note that, for each $W \in \mathcal{W}_{L}^{r, \tau}, \tau>0$, the foliation $T^{n} W$ converges to the invariant foliation (since the contraction of the cone fields implies that, for all $x \in M$, $D_{T^{n}{ }_{x}} T^{-n} \mathcal{T}_{T^{n}{ }_{x}} W\left(T^{n} x\right)$ converges to the stable distribution $E^{s}$ ). (Here $\mathcal{T}_{x} V$ is the tangent space of the manifold $V$ at the point $x$ and $W(x)$ is the fiber of the foliation passing through $x$, while $E^{s}(x)$ is the stable subspace in $\mathcal{T}_{x} M$.) Moreover, if $\mathbb{F}_{n}$ describes $T^{n} W$, then the $\partial_{y}^{\alpha} \mathbb{F}_{n}$ are uniformly Hölder. Accordingly, for each $\tau^{\prime}<\tau$, by compactness, $T^{n} W$ has a convergent subsequence, and hence it converges to the stable foliation, and all the quantities in the definition of $\mathcal{W}_{L}^{r, \tau}$ converge as well. It follows that the stable foliations have $\mathcal{C}^{r}$ leaves with derivatives in $y$ that are uniformly $\tau^{\prime}$ Hölder in $x$. Analogously, $H^{F}$ and its derivatives converge. This implies that the invariant foliation has a holonomy that is uniformly absolutely continuous (see Lemma B.7 and Remark B.8 for the definitions 
of the holonomy, its Jacobian $J^{F}$ and its properties). Similar results hold also in the case when $\tau=0$, but the argument is a bit more involved.

Proof of Proposition B.1. The first step in proving the proposition is to determine, for each $\xi \in M$ and $n \in \mathbb{N}$, the functions $F_{T^{-n} \xi}^{n}$ associated to $W^{n}$. (Since the point $\xi$ in the present argument is fixed once and for all, in the following, we will often suppress the subscript $\xi$. We will also suppress the $n$ dependence if no confusion arises.) Note that it suffices to compute the norms in (B.2) in a special neighborhood of $T^{-n} \xi=: \xi^{\prime}$. Indeed, if $\phi_{j}\left(\xi^{\prime}\right)=\left(x^{\prime}, y^{\prime}\right)$ and $\widehat{U}_{u}^{0}=\left\{x \in \mathbb{R}^{d_{u}}:\|x\| \leq \delta_{\star}\right\}$, then it suffices to consider the set $\left(x^{\prime}, y^{\prime}\right)+\widehat{U}_{u}^{0} \times U_{s}^{0}$ since, setting $\zeta_{\xi^{\prime}, x}=\phi_{j}^{-1}\left(x^{\prime}+x, y^{\prime}\right)$, a direct computation shows that $F_{\xi_{\xi^{\prime}, x}}(u, y)=F_{\xi^{\prime}}(x+u, y)-x$. Thus

$$
\sup _{\substack{x \in U_{u}^{0} \\\|x-\tilde{x}\| \leq \delta_{\star}}} \frac{\left\|F_{\xi^{\prime}}(x, y)-F_{\xi^{\prime}}(\tilde{x}, y)\right\|}{\|x-\tilde{x}\|^{\tau}}=\sup _{x \in U_{u}^{0}} \sup _{u \in \widehat{U}_{u}^{0}} \frac{\left\|F_{\zeta_{\xi^{\prime}, x}}(0, y)-F_{\zeta_{\xi^{\prime}, x}}(u, y)\right\|}{\|u\|^{\tau}} .
$$

While, for $|\alpha|>0$,

$$
\begin{aligned}
& \left\|\partial_{y}^{\alpha} F_{\xi^{\prime}}(\cdot, y)\right\|\left\|_{\mathcal{C}^{\tau}\left(U_{u}^{0}, \mathbb{R}^{d_{u}}\right)}=\sup _{x \in U_{u}^{0}}\right\| \partial_{y}^{\alpha} F_{\xi^{\prime}}(x, y) \|+\sup _{\substack{x \in U_{u}^{0} \\
\|x-\tilde{x}\| \leq \delta_{\star}}} \frac{\left\|\partial_{y}^{\alpha} F_{\xi^{\prime}}(x, y)-\partial_{y}^{\alpha} F_{\xi^{\prime}}(\tilde{x}, y)\right\|}{\|x-\tilde{x}\|^{\tau}} \\
& \quad=\sup _{x \in U_{u}^{0}}\left\{\left\|\partial_{y}^{\alpha} F_{\zeta_{\xi^{\prime}, x}}(0, y)\right\|+\sup _{u \in \widehat{U}_{u}^{0}} \frac{\left\|\partial_{y}^{\alpha} F_{\zeta_{\xi^{\prime}, x}}(0, y)-\partial_{y}^{\alpha} F_{\zeta_{\xi^{\prime}, x}}(u, y)\right\|}{\|u\|^{\tau}}\right\} .
\end{aligned}
$$

Hence, the sup on $y$ and $\xi^{\prime}$ can be computed by taking the sup of the quantity in the curly bracket (and the same for $H^{F}$ ).

Let $\left(V_{i}, \phi_{i}\right),\left(V_{j}, \phi_{j}\right)$ be the charts associated to $\xi$ and $T^{-n} \xi$, respectively, and consider the map $S=\phi_{j} \circ T^{-n} \circ \phi_{i}^{-1}$. By a simple translation, we can assume, without loss of generality, that $\phi_{i}(\xi)=0$ and $\phi_{j}\left(T^{-n} \xi\right)=0$. From now on, we use $(x, y)$ for the coordinate names at $\phi_{i}(\xi)$ and $(u, s)$ for the coordinate names at $\phi_{j}\left(T^{-n} \xi\right)$. By a linear change of coordinates, that leaves $\{y=0\}$ and $\{s=0\}$ fixed, and we can have $\partial_{y} F(0,0)=$ $\partial_{s} F^{n}(0,0)=0$. Such a change of coordinates may affect the norms and yield some extra (uniformly bounded) constant in the estimates. We will ignore this to simplify the notation since its effect is trivial. Also, remember that, by construction, $F(x, 0)=x, F^{n}(u, 0)=u$.

It follows from the usual graph transform (see [KH, Proof of Theorem 6.2.8 (Hadamard-Perron)]) that

$$
S^{-1}(u, 0)=(\beta(u), G(\beta(u)),
$$

where $\beta \in \mathcal{C}^{r+\tau}\left(\mathbb{R}^{d_{u}}, \mathbb{R}^{d_{u}}\right)$ and $\|G\|_{\mathcal{C}^{r+\tau}\left(\mathbb{R}^{d_{u}}, \mathbb{R}^{d_{s}}\right)} \leq c_{1}$ for some $c_{1}>0$ depending only on $T$. Moreover, $\|D G\| \leq \eta<1$ by the invariance of the cone field and $\beta(0)=0, G(0)=0$ by construction. Moreover, by (2.2), $\left\|(D \beta)^{-1}\right\|_{\mathcal{C}^{0}} \leq c_{0}^{-1} \lambda^{-n}$ while, setting $\lambda_{+}=$ $\max \left\{\|D T\|,\left\|D T^{-1}\right\|\right\}$, we have, for some constant $C_{1}>0$,

$$
\|\beta(u)\| \leq\|(\beta(u), G \circ \beta(u))\| \leq C_{1} \lambda_{+}^{n}\|u\| .
$$

In addition, $\left\{\left(z, D_{\xi} G(z)\right)\right\}_{z \in \mathbb{R}^{d_{u}}}$ is uniformly traversal to $\left\{\left(D_{\xi} F(\zeta), \zeta\right)\right\}_{\zeta \in \mathbb{R}^{d_{s}}}$. 
Hence, setting

$$
D_{(x, y)} S=\left(\begin{array}{ll}
A & B \\
C & E
\end{array}\right)
$$

we have

$$
\begin{aligned}
& A(x, G(x))=\left(D_{x} \beta\right)^{-1}-B(x, G(x)) D_{x} G, \\
& C(x, G(x))=-E(x, G(x)) D_{x} G ; \quad B(0,0)=0,
\end{aligned}
$$

where the last equality follows by the choice of the coordinates.

For each $x$, the manifold $\{(F(x, y), y)\}_{y \in \mathbb{R}^{d_{s}}}$ intersects the manifold $\{(z, G(z))\}_{\mathbb{R}^{d_{u}}}$ in a unique point determined by the equation

$$
(F(x, y), y)=(z, G(z)),
$$

which is equivalent to $L(z, x):=z-F(x, G(z))=0$. Since $L(0,0)=0$, we apply the implicit function theorem and obtain a function $\Gamma: U_{u}^{0} \subset \mathbb{R}^{d_{u}} \rightarrow \mathbb{R}^{d_{u}}$ such that

$$
\Gamma(x)=F(x, G \circ \Gamma(x)) .
$$

Note that the implicit function theorem yields a uniform domain $D(\Gamma)$, of $\Gamma$. Hence, we can take $\delta_{0}$ small enough so that $D(\Gamma) \supset U_{u}^{0}$.

Since $\left\|\partial_{y} F\right\| \leq 1$ and recalling that $\|D G\| \leq \eta<1, \mathbb{1}-\partial_{y} F D G$ is invertible. Hence,

$$
D \Gamma=\left(\mathbb{1}-\partial_{y} F D G\right)^{-1} \partial_{x} F .
$$

Note that, remembering (2.5), $D_{0} \Gamma=\mathbb{1}$. Moreover, $\Gamma$ is invertible since, recalling (B.7), $\Gamma(x)=\Gamma\left(x^{\prime}\right)$ implies that

$$
\begin{aligned}
(F(x, G \circ \Gamma(x)), G \circ \Gamma(x)) & =\left(F\left(x^{\prime}, G \circ \Gamma\left(x^{\prime}\right)\right), G \circ \Gamma(x)\right) \\
& =\left(F\left(x^{\prime}, G \circ \Gamma(x)\right), G \circ \Gamma(x)\right),
\end{aligned}
$$

which forces $x=x^{\prime}$ since the leaves of the foliation are disjoint by hypothesis.

By definition, for each $u \in \mathbb{R}^{d_{u}}$ small enough, $\left\{\left(F^{n}(u, s), s\right)\right\}_{s \in \mathbb{R}^{d_{s}}}$ is the graph of the leaf of $W^{n}$ passing through $(u, 0)$, and hence of the image of the leaf of $W$ passing through $\left(\Gamma^{-1} \circ \beta(u), 0\right)$. In other words, $\left\{\left(F^{n}(u, s), s\right)\right\}_{s \in \mathbb{R}^{d_{s}}}$ coincides with the leaf $\left\{S\left(F\left(\Gamma^{-1} \circ\right.\right.\right.$ $\beta(u), y), y)\}_{y \in \mathbb{R}^{d_{s}}}$.

To continue, we need some estimates on $D S$. But, before that, it is convenient to make some choices and definitions whose meaning will become clear later in the proof. Let $\tau_{0} \in(0,1)$ be such that

$$
\sigma_{1}:=\max \left\{v, \lambda^{-1}\right\} \cdot \lambda_{+}^{8 \tau_{0}}<1 .
$$

Next, let $\sigma_{1}<\sigma<1$, fix $C_{\star}>0$ to be chosen later (see equations (B.16), (B.20) and (B.25)) and let $n_{\star}$ be the smallest integer such that

$$
C_{\star} \sigma_{1}^{n_{\star}}=\sigma^{n_{\star}}<(1 / 8)^{r}, \quad v^{n_{\star}} \leq 2^{-2 r} .
$$

Remark B.4. Up to now, $\delta_{0}$ was arbitrary provided we chose it small enough: the requirements are in $\$ 2.4$, where we fix the charts just after (B.7). In the following, we will have also a condition in equation (B.12) to apply the implicit function theorem, and we will use $\delta_{0}<1 / 8$ in equation (B.14). All such choices can be summarized by the condition 
$\delta_{0} \leq \delta_{1}$ for some $\delta_{1} \in(0,1 / 8)$ depending only on $T$. However, in the next lemma, we will have a requirement depending on $n$.

LEMMA B.5. There exists $\eta, \sigma_{0} \in(0,1)$ and $C_{0} \geq \max \left\{2,6 c_{0}^{-1}\right\}$ such that, for each $n \in$ $\mathbb{N}, d_{u} \times d_{s}$ matrix $U,\|U\| \leq 1$,

$$
\|C U+E\| \geq C_{0}^{-1} v^{-n} ; \quad\left\|E^{-1} C\right\| \leq \eta ; \quad\left\|(A U+B)(C U+E)^{-1}\right\| \leq \eta .
$$

In particular, $\left\|E^{-1}\right\| \leq C_{0} v^{n}$. Moreover, there exists a constant $C_{b}>0$ such that if we choose $\delta_{0}=\min \left\{\delta_{1}, C_{0} C_{b}^{-1} \lambda_{+}^{-4 n_{\star}} / 3\right\}$, then, for all $n \in\left\{n_{\star}, \ldots, 2 n_{\star}\right\},\|A\|+\|B\| \leq$ $C_{0} \lambda^{-n}$.

Proof. By the strict invariance of the cone field (see §2.2) and the Anosov property (2.2), it follows that, for each $U$, there exist matrices $U_{1}, H$, with $\left\|U_{1}\right\| \leq \eta$ and $\|H\| \geq$ $\left(c_{0} \sqrt{2}\right) /\left(\sqrt{1+\eta^{2}}\right) v^{-n}$, such that

$$
\left(U_{1} H v, H v\right)=D S\left(\begin{array}{c}
U v \\
v
\end{array}\right)=([A U+B] v,[C U+E] v) .
$$

Thus, $H=C U+E$ and $U_{1}=(A U+B)(C U+E)^{-1}$ from which the first and third inequalities readily follow. Analogously, for each $V$, there exists $\tilde{V}, \tilde{H},\|\tilde{V}\| \leq \eta,\|\tilde{H}\| \leq$ $c_{0}^{-1} \sqrt{2} \lambda^{-n}$, such that

$$
D S\left(\begin{array}{c}
v \\
\tilde{V} v
\end{array}\right)=(\tilde{H} v, V \tilde{H} v)
$$

which implies the second inequality and, for $V=0$, yields $\left\|E^{-1} C\right\|=\|\tilde{V}\| \leq \eta$. Note that

$$
\begin{aligned}
\partial_{x_{p}} D & T^{-n}=D\left(D_{T^{-n+1} x} T^{-1} \cdots D_{x} T^{-1}\right) \\
= & \sum_{k=0}^{n-1} \sum_{j=1}^{d} D_{T^{-n+1} x} T^{-1} \cdots D_{T^{-k-1} x} T^{-1} \partial_{x_{j}}\left(D_{T^{-k} x_{x}} T^{-1}\right) D_{T^{-k+1} x_{x}} T^{-1} \cdots D_{x} T^{-1} \\
& \quad \times\left(D_{x} T^{-k}\right)_{j p} .
\end{aligned}
$$

Recalling that $\lambda_{+}=\max \left\{\|D T\|,\left\|D T^{-1}\right\|\right\}$, it follows that there exists a constant $C_{b}>0$, depending only on $T$ and on the coordinate changes $\left\{\phi_{i}\right\}$, such that $\|D B\| \leq C_{b} \lambda_{+}^{2 n_{\star}}$. Then, since $B(0,0)=0$ (see (B.6)), it follows that

$$
\|B\| \leq \delta_{0}\|D B\| \leq C_{0} \lambda_{+}^{-2 n_{\star}} / 3 \leq C_{0} \lambda^{-n} / 3
$$

and

$$
\|A\| \leq\|\tilde{H}\|+\|B \tilde{V}\| \leq\|\tilde{H}\|+\eta C_{0} \lambda^{-n} / 3 \leq\left(c_{0}^{-1} \sqrt{2} \lambda^{-n}+C_{0} \lambda^{-n} / 3\right) \leq \frac{2 C_{0}}{3} \lambda^{-n},
$$

from which the last assertion of the lemma readily follows.

We are now ready to study $F^{n}$. Let us consider the function

$$
\Xi(v, s, u, y)=(v, s)-S\left(F\left(\Gamma^{-1} \circ \beta(u), y\right), y\right) .
$$


It is convenient to set $\Upsilon(u)=\Gamma^{-1} \circ \beta(u)$. Note that (B.7) implies that

$$
\|x\|=\left\|F\left(\Gamma^{-1}(x), G(x)\right)\right\| \geq\left\|F\left(\Gamma^{-1}(x), 0\right)\right\|-\eta\|x\|=\left\|\Gamma^{-1}(x)\right\|-\eta\|x\|,
$$

that is

$$
\left\|\Gamma^{-1}(x)\right\| \leq(1+\eta)\|x\| .
$$

We want to ensure that $\|\Upsilon(u)\| \leq \delta_{0}$. Recalling (B.4), this is implied by

$$
C_{1}(1+\eta) \delta_{\star} \lambda_{+}^{2 n_{\star}} \leq \delta_{0} .
$$

Note that

$$
\begin{aligned}
\Xi(u, 0, u, G(\beta(u))) & =(u, 0)-S(F(\Upsilon(u), G(\beta(u))), G(\beta(u))) \\
& =(u, 0)-S(\beta(u), G(\beta(u)))=0 .
\end{aligned}
$$

To study the zeros of $\Xi$, we apply the implicit function theorem. Since

$$
\operatorname{det}\left(\begin{array}{ll}
\partial_{v} \Xi & \partial_{y} \Xi
\end{array}\right)=\operatorname{det}\left(\begin{array}{ll}
\mathbb{1} & A \partial_{y} F+B \\
0 & C \partial_{y} F+E
\end{array}\right)
$$

we can compute

$$
\begin{aligned}
& \operatorname{det}\left(\partial_{v} \Xi(u, 0, u, G(\beta(u))) \quad \partial_{y} \Xi(u, 0, u, G(\beta(u)))\right) \\
& =\operatorname{det}(E(\beta(u), G(\beta(u)))) \operatorname{det}\left(\mathbb{1}-D G(\beta(u)) \partial_{y} F(\Upsilon(u))\right) \neq 0,
\end{aligned}
$$

where we have used (B.6) and $\left\|D G \partial_{y} F\right\| \leq \eta$. Thus there exists a uniform (in $n$ ) neighborhood of $(u, G(\beta(u))$ where the implicit function theorem can be applied. Thus, we can choose $\delta_{0}$ small enough so that, for each $n \in \mathbb{N}$, there exist $F^{n}, \Phi \in \mathcal{C}^{r}$, and

$$
\begin{aligned}
& v=F^{n}(u, s), \\
& y=\Phi(u, s) .
\end{aligned}
$$

Moreover, defining the change of coordinates $\Omega(u, s)=\left(\Gamma^{-1} \circ \beta(u), \Phi(u, s)\right)=(x, y)$,

$$
\mathbb{F}^{n}(u, s)=S \circ \mathbb{F} \circ \Omega(u, s) .
$$

Note that

$$
\left\|\mathbb{F}^{n}(u, s)\right\| \leq\left\|\mathbb{F}^{n}(u, s)-\mathbb{F}^{n}(u, 0)\right\|+\left\|\mathbb{F}^{n}(u, 0)\right\| \leq\|s\|+\|u\| \leq 2 \delta_{0} \leq \frac{1}{4} .
$$

Differentiating (B.13) with respect to $s$, we obtain

$$
\left(\begin{array}{cc}
\partial_{u} F^{n} & \partial_{s} F^{n} \\
0 & \mathbb{1}
\end{array}\right)=\left(\begin{array}{cc}
A & B \\
C & E
\end{array}\right)\left(\begin{array}{cc}
\partial_{x} F & \partial_{y} F \\
0 & \mathbb{1}
\end{array}\right)\left(\begin{array}{cc}
\partial_{u} \Upsilon & 0 \\
\partial_{u} \Phi & \partial_{s} \Phi
\end{array}\right)
$$

which yields

$$
\begin{aligned}
\partial_{s} \Phi & =\left(E \circ \mathbb{F} \circ \Omega+C \circ \mathbb{F} \circ \Omega \cdot \partial_{y} F \circ \Omega\right)^{-1}, \\
\partial_{s} F^{n} & =\left(A \circ \mathbb{F} \circ \Omega \cdot \partial_{y} F \circ \Omega+B \circ \mathbb{F} \circ \Omega\right) \partial_{s} \Phi .
\end{aligned}
$$


Then, provided $C_{\star} \geq\left(C_{0}^{2} /(1-\eta)\right)$, Lemma B.5 yields

$$
\begin{aligned}
\left\|\partial_{s} \Phi\right\| & \leq\left\|E^{-1}\right\|\left\|\left(\mathbb{1}+\left[E^{-1} C\right] \circ \mathbb{F} \cdot \partial F\right)^{-1}\right\| \leq \frac{C_{0}}{1-\eta} v^{n} \leq \frac{1}{8^{r}}, \\
\left\|\partial_{S} F^{n}\right\| & \leq \frac{C_{0}^{2}}{1-\eta} v^{n} \lambda^{-n} \leq \frac{1}{8^{r}} .
\end{aligned}
$$

We now study $\partial_{s}^{\alpha} F^{n}$ when $|\alpha| \geq 2$. Differentiating (B.15) and setting $\Delta_{-}=(\mathbb{1}+$ $\left.\left(E^{-1} C\right) \circ \mathbb{F} \cdot \partial_{y} F\right)^{-1}$ and $\Delta_{+}=\left(A \circ \mathbb{F} \cdot \partial_{y} F+B \circ \mathbb{F}\right)$, we obtain

$$
\begin{aligned}
\partial_{s_{2}} \partial_{s_{1}} F^{n}= & \sum_{i_{1}, i_{2}=1}^{d_{s}} \mathcal{H}\left(D S \circ F, \partial_{y} F, \partial_{y_{i_{2}}} \partial_{y_{i_{1}}} F\right) \circ \Omega \cdot \partial_{s_{1}} \Phi_{i_{1}} \partial_{s_{2}} \Phi_{i_{2}} \\
& +\Theta_{1}\left(D S \circ F, D^{2} S \circ F, \partial_{y} F\right) \circ \Omega,
\end{aligned}
$$

where $\Theta_{1}$ is a rational function of its arguments and, for an arbitrary matrix $\mathcal{R}$,

$$
\begin{aligned}
\mathcal{H}\left(D S \circ F, \partial_{y} F, \mathcal{R}\right):= & \left\{A \circ \mathbb{F} \cdot \mathcal{R} \cdot \Delta_{-} \cdot E^{-1} \circ \mathbb{F}\right\} \\
& -\left\{\Delta_{+} \Delta_{-} \cdot\left(E^{-1} C\right) \circ \mathbb{F} \cdot \mathcal{R} \cdot \Delta_{-} \cdot E^{-1} \circ \mathbb{F}\right\} .
\end{aligned}
$$

Note that Lemma B.5 implies that

$$
\left\|\mathcal{H}\left(D S \circ F, \partial_{y} F, \mathcal{R}\right)\right\| \leq \frac{C_{0}^{2}}{(1-\eta)^{2}} \lambda^{-n} v^{n}\|\mathcal{R}\| .
$$

Differentiating further (B.17) we can prove, by induction, that, for all $l \leq r$,

$$
\begin{aligned}
\partial_{s_{j_{l}}} \cdots \partial_{s_{j_{1}}} F^{n}= & \sum_{i_{1}, \ldots, i_{l}=1}^{d_{s}} \mathcal{H}\left(D S \circ F, \partial_{y} F, \partial_{y_{i_{l}}} \cdots \partial_{y_{i_{1}}} F\right) \circ \Omega \cdot \partial_{s_{j_{1}}} \Phi_{i_{1}} \cdots \partial_{s_{j_{l}}} \Phi_{i_{l}} \\
& +\Theta_{l-1}\left(D S \circ F, \ldots, D^{l} S \circ F, \partial_{y} F, \ldots, \partial_{y}^{l-1} F\right) \circ \Omega, \quad \text { (B. }
\end{aligned}
$$

where the $\Theta_{l}$ are sums of functions $k_{j}$-multilinear in $\partial_{y}^{j} F$, for $j \in\{2, \ldots, l\}$, such that $\sum_{j=2}^{l} k_{j}(j-1) \leq l$. Indeed, we have seen that this is true for $l=2$. On the other hand, if it is true for $l-1$, then differentiating (B.19) we produce several terms. Let us analyze them one by one. The term proportional to $\mathcal{H}$, when differentiated with respect to $\partial_{y}^{l} F$, yields the correct term proportional of $\mathcal{H}$. When differentiated with respect to $D^{k} S \circ F$, it yields a function of $D^{k+1} S \circ F$ multiplied by $\partial_{y} F \cdot \partial_{s} \Phi$ so the multilinearity with respect to $\partial_{y}^{j} F$, for $j \in\{2, \ldots, l\}$, is unchanged. When differentiating with respect to $\partial_{y} F$, the term gets multiplied by $\partial_{y}^{2} F$. (Recall that a dependence from $\partial_{y} F$ is contained in $\partial_{s} \Phi$ (see (B.15)).) Thus, calling $k_{j}^{\prime}$ the multilinearities of the term obtained, we have $k_{l}^{\prime}=1, k_{2}^{\prime}=1$ and all the other $k_{j}^{\prime}$ are zero: that is, $\sum_{j=2}^{l} k_{j}^{\prime}(j-1)=l-1+1=l$.

Next we must differentiate $\Theta_{l-1}$. Again the only change in the multilinearity occurs when differentiating with respect to a $\partial_{y}^{m} F, m \in\{1, \ldots, l-1\}$. If $j=2$, then we have (again, calling the new multilinearities $k^{\prime}$ ) $k_{2}^{\prime}=k_{2}+1$ and $k_{j}^{\prime}=k_{j}$ for $j>2$ : that is, $\sum_{=2}^{l-1} k_{j}^{\prime}(j-1)=l-1+1=l$. If $m>1$, then $k_{m}^{\prime}=k_{m}-1, k_{m+1}^{\prime}=k_{m+1}+1$ and $k_{j}^{\prime}=k_{j}$ for $j \notin\{m, m+1\}$ : that is, $\sum_{j=2}^{l} k_{j}^{\prime}(j-1)=l-1-(m-1)+m=l$, which proves our claim. 
Using equations (B.16) and (B.18) to estimate (B.19) yields, for all $l \in\{2, \ldots, r\}$,

$$
\begin{aligned}
\left\|\partial_{s}^{l} F^{n}\right\| & \leq d_{s}^{l} \frac{C_{0}^{2+l}}{(1-\eta)^{2+l}} \lambda^{-n} v^{n(l+1)}\left\|\partial_{s}^{l} F\right\|+\left\|\Theta_{l-1}\right\|_{\infty} \\
& \leq d_{s}^{l} \frac{C_{0}^{2+l}}{(1-\eta)^{2+l}} \lambda^{-n} v^{n(l+1)} L_{1}^{(l-1)^{2}}+C_{n} L_{1}^{\sum_{j=2}^{l-1} k_{j}(j-1)^{2}} \\
& \leq\left[d_{s}^{l} \frac{C_{0}^{2+l}}{(1-\eta)^{2+l}} \lambda^{-n} v^{n(l+1)}+C_{n} L_{1}^{-(l-1)}\right] L_{1}^{(l-1)^{2}}
\end{aligned}
$$

Choosing

$$
\begin{aligned}
C_{\star} & \geq d_{s}^{r} \frac{C_{0}^{2+r}}{(1-\eta)^{2+r}}, \\
L_{1} & >\max \left\{1,2^{r^{2}} \max \left\{C_{n_{\star}}, \ldots, C_{2 n_{\star}}\right\}\right\},
\end{aligned}
$$

equation (B.9) implies, as claimed, that

$$
\left\|\partial_{S}^{l} F^{n}\right\| \leq\left[L_{1} / 2\right]^{(l-1)^{2}} .
$$

Next, we estimate the Hölder norms of $\partial_{s}^{\alpha} \mathbb{F}^{n}$ for $|\alpha| \leq r-1$. We first treat the case $|\alpha|=0$. By strict cone field invariance and the continuity of the cone field, it follows that, for all $s$,

$$
S^{-1} \mathbb{F}^{n}(u, s)=\left(F \circ \Omega(u, s), G_{s}(F \circ \Omega(u, s))\right)
$$

with $\left\|D G_{s}\right\| \leq \eta<1$. Notice that (B.13), (B.22) and (B.3) imply that

$$
\begin{aligned}
(F \circ \Omega(u, 0), \Phi(u, 0)) & =\left(F \circ \Omega(u, 0), G_{0}(F \circ \Omega(u, 0))\right) \\
& =S^{-1} \mathbb{F}^{n}(u, 0)=S^{-1}(u, 0)=(\beta(u), G(\beta(u))),
\end{aligned}
$$

that is $G_{0}=G$ and

$$
\Phi(u, 0)=G(\beta(u)) .
$$

Analogously, by (B.13), $S^{-1} \mathbb{F}^{n}(u, s)=(F \circ \Omega(u, s), \Phi(u, s))$. Hence

$$
\begin{aligned}
& \left\|\Phi(u, s)-\Phi\left(u^{\prime}, s\right)\right\|=\left\|G_{s}(F \circ \Omega(u, s))-G_{s}\left(F \circ \Omega\left(u^{\prime}, s\right)\right)\right\| \\
& \quad \leq \eta\left\|F \circ \Omega(u, s)-F \circ \Omega\left(u^{\prime}, s\right)\right\| \\
& \quad \leq \eta\left\|F(\Upsilon(u), \Phi(u, s))-F\left(\Upsilon\left(u^{\prime}\right), \Phi(u, s)\right)\right\|+\eta\left\|\Phi(u, s)-\Phi\left(u^{\prime}, s\right)\right\|,
\end{aligned}
$$

where we have used $\left\|\partial_{y} F\right\| \leq 1$. Accordingly,

$$
\left\|\Phi(u, s)-\Phi\left(u^{\prime}, s\right)\right\| \leq \frac{\eta}{1-\eta}\left\|F(\Upsilon(u), \Phi(u, s))-F\left(\Upsilon\left(u^{\prime}\right), \Phi(u, s)\right)\right\| .
$$

Next, we prove an auxiliary lemma, which will be used repeatedly in the following.

Lemma B.6. Let $\mathcal{G}: U^{0} \rightarrow \mathbb{R}^{d^{\prime}}, d^{\prime} \in \mathbb{N}$. Assume that $\sup _{y \in U_{s}^{0}}\|\mathcal{G}(\cdot, y)\|_{\mathcal{C}^{0}\left(U_{u}^{0}, \mathbb{R}^{d^{\prime}}\right)} \leq D_{0}$, $\sup _{y \in U_{s}^{0}}\|\mathcal{G}(\cdot, y)\|_{\mathcal{C}^{\tau}\left(U_{u}^{0}, \mathbb{R}^{d^{\prime}}\right)} \leq D^{\prime}$ with $\tau \in\left[0, \tau_{0}\right]$ and $\left\|\partial_{y} \mathcal{G}\right\|_{\mathcal{C}^{0}} \leq \tilde{D}$. Then, for all $n \in$ $\left\{n_{\star}, \ldots, 2 n_{\star}\right\}$,

$c_{0}^{-1} \max \left\{v^{n}, \lambda^{-n}\right\}\|\mathcal{G} \circ \Omega(u, s)-\mathcal{G} \circ \Omega(0, s)\| \leq \frac{1}{8}\left[(1-\eta) \max \left\{D_{0}, D^{\prime}\right\}+2 \eta \tilde{D}\right]\|u\|^{\tau}$. 
Proof. Let $\theta=\max \left\{v, \lambda^{-1}\right\}$. We start by analyzing $\|\Upsilon(u)\| \leq \delta_{\star}$. By (B.24), we get

$$
\|\Phi(u, s)-\Phi(0, s)\| \leq \frac{2 \eta}{(1-\eta)}\|\Upsilon(u)\|^{\tau_{0}} .
$$

Hence, by (B.10) and (B.4),

$$
\begin{gathered}
c_{0}^{-1} \theta^{n}\|\mathcal{G} \circ \Omega(u, s)-\mathcal{G} \circ \Omega(0, s)\| \leq c_{0}^{-1} \theta^{n}\left[D^{\prime}\|\Upsilon(u)\|^{\tau}+\tilde{D}\|\Phi(u, s)-\Phi(0, s)\|\right] \\
\leq \frac{\theta^{n}(1+\eta)^{\tau}}{c_{0}}\left[D^{\prime}+\frac{2 \tilde{D} \eta}{1-\eta}\right]\|\beta(u)\|^{\tau} \leq \frac{\theta^{n}(1+\eta)^{\tau}}{c_{0}}\left[D^{\prime}+\frac{2 \tilde{D} \eta}{1-\eta}\right] C_{1}^{\tau} \lambda_{+}^{\tau n}\|u\|^{\tau} .
\end{gathered}
$$

Consequently, provided that $C_{\star}$ in (B.9) satisfies (see Remark B.4 for the definition of $\delta_{1}$; also we consider a $C_{\star}$ larger than what is needed at this stage for later purposes in this proof)

$$
C_{\star} \geq \frac{2(1+\eta)^{\tau} C_{1}^{\tau}\left(\delta_{1}^{-\tau_{0}}+C_{b}^{\tau}\right)}{c_{0}(1-\eta)},
$$

we have

$$
\begin{aligned}
c_{0}^{-1} \theta^{n}\|\mathcal{G} \circ \Omega(u, s)-\mathcal{G} \circ \Omega(0, s)\| & \leq\left[C_{\star}(1-\eta) D^{\prime}+2 \eta C_{\star} \tilde{D}\right] \sigma_{1}^{n}\|u\|^{\tau} \\
& \leq \frac{1}{8}\left[(1-\eta) D^{\prime}+2 \eta \tilde{D}\right]\|u\|^{\tau},
\end{aligned}
$$

where, in the last line, we have used (B.9). We are left with the analysis of the case when $\|\Upsilon(u)\| \geq \delta_{\star}$. By (B.10), (B.11), (B.4) and (B.8),

$$
\begin{aligned}
c_{0}^{-1} \theta^{n}\|\mathcal{G} \circ \Omega(u, s)-\mathcal{G} \circ \Omega(0, s)\| & \leq c_{0}^{-1} \theta^{n} 2 D_{0} \leq c_{0}^{-1} \theta^{n}(1+\eta)^{\tau} 2 D_{0} \delta_{\star}^{-\tau}\|\beta(u)\|^{\tau} \\
& \leq c_{0}^{-1} \theta^{n}(1+\eta)^{\tau} 2 D_{0} \delta_{0}^{-\tau} \lambda_{+}^{2 \tau n_{\star}} C_{1}^{\tau} \lambda_{+}^{\tau n}\|u\|^{\tau} .
\end{aligned}
$$

Thus, if $\delta_{0}=\delta_{1}$, then

$$
c_{0}^{-1} \theta^{n}\|\mathcal{G} \circ \Omega(u, s)-\mathcal{G} \circ \Omega(0, s)\| \leq \frac{(1-\eta) D_{0}}{8}\|u\|^{\tau} .
$$

Otherwise, recalling Lemma B.5 and equation (B.9),

$$
\begin{aligned}
c_{0}^{-1} \theta^{n}\|\mathcal{G} \circ \Omega(u, s)-\mathcal{G} \circ \Omega(0, s)\| & \leq \frac{(1-\eta) 3^{\tau} C_{b}^{\tau} C_{\star}}{C_{0}^{\tau}} \delta_{0}^{-\tau} \theta^{n_{\star}} \lambda_{+}^{4 n_{\star} \tau} D_{0}\|u\|^{\tau} \\
& \leq(1-\eta) C_{\star} \theta^{n_{\star}} \lambda_{+}^{8 n_{\star} \tau} D_{0}\|u\|^{\tau} \leq \frac{(1-\eta) D_{0}}{8}\|u\|^{\tau},
\end{aligned}
$$

from which the lemma follows.

Note, for future use, that the above computation also implies that

$$
c_{0}^{-1} \theta^{n}\|\Phi(u, s)-\Phi(0, s)\| \leq \frac{1}{2}\|u\|^{\tau_{0}} .
$$

We now estimate $\left\|\mathbb{F}^{n}(u, s)-\mathbb{F}^{n}(0, s)\right\|$. By (2.2) and (B.13),

$$
\left\|\mathbb{F}^{n}(u, s)-\mathbb{F}^{n}(0, s)\right\| \leq c_{0}^{-1} \lambda^{-n}\|(F \circ \Omega(u, s), \Phi(u, s))-(F \circ \Omega(0, s), \Phi(0, s))\| .
$$


Recalling (B.28), we can apply Lemma B.6 with $\mathcal{G}=F, \tau=\tau_{0}, D_{0}=1, D^{\prime}=2$ and $\tilde{D}=1$ to obtain, for all $n \in\left[n_{\star}, 2 n_{\star}\right]$,

$$
\left\|\mathbb{F}^{n}(u, s)-\mathbb{F}^{n}(0, s)\right\| \leq \frac{1}{4}\|u\|^{\tau_{0}} .
$$

From this, and recalling (B.14), the required estimate follows: that is,

$$
\sup _{s}\left\|\mathbb{F}^{n}(\cdot, s)\right\|_{\mathcal{C}^{\tau_{0}}} \leq \frac{1}{2}
$$

Next, we discuss the case when $|\alpha|>0$. We start by estimating $\left\|\partial_{s} \Phi\right\|_{\mathcal{C}_{0}^{\tau}}$. To simplify the notation in the expression below, let $a=(u, s)$ and $b=(0, s)$. Using (B.15), (B.16) and Lemma B.5, notice that

$$
\begin{aligned}
\| \partial_{s} \Phi & (a)-\partial_{s} \Phi(b)\|\leq\| E^{-1} \circ \mathbb{F} \circ \Omega(b) \\
& -E^{-1} \circ \mathbb{F} \circ \Omega(a)\|\cdot\|\left(\mathbb{1}+E \circ \mathbb{F} \circ \Omega(b)^{-1} C \circ \mathbb{F} \circ \Omega(b) \cdot \partial_{y} F \circ \Omega(b)\right)^{-1} \| \\
& +\left\|\left(E^{-1} \circ \mathbb{F} \circ \Omega(a)\right)\right\| \cdot\left\|\left(\mathbb{1}+E \circ \mathbb{F} \circ \Omega(a)^{-1} C \circ \mathbb{F} \circ \Omega(a) \cdot \partial_{y} F \circ \Omega(a)\right)^{-1}\right\| \\
& \times \| E \circ \mathbb{F} \circ \Omega(a)^{-1} C \circ \mathbb{F} \circ \Omega(a) \cdot \partial_{y} F \circ \Omega(a) \\
& -E \circ \mathbb{F} \circ \Omega(b)^{-1} C \circ \mathbb{F} \circ \Omega(b) \cdot \partial_{y} F \circ \Omega(b) \| \\
& \times\left\|\left(\mathbb{1}+E \circ \mathbb{F} \circ \Omega(b)^{-1} C \circ \mathbb{F} \circ \Omega(b) \cdot \partial_{y} F \circ \Omega(b)\right)^{-1}\right\| \\
\leq & \left\|E^{-1} \circ \mathbb{F} \circ \Omega(b)-E^{-1} \circ \mathbb{F} \circ \Omega(a)\right\| \\
& \times\left\|\left(\mathbb{1}+E \circ \mathbb{F} \circ \Omega(b)^{-1} C \circ \mathbb{F} \circ \Omega(b) \cdot \partial_{y} F \circ \Omega(b)\right)^{-1}\right\| \\
& +\frac{C_{0} v^{n}}{1-\eta} \| E \circ \mathbb{F} \circ \Omega(a)^{-1} C \circ \mathbb{F} \circ \Omega(a) \cdot \partial_{y} F \circ \Omega(a) \\
& -E \circ \mathbb{F} \circ \Omega(b)^{-1} C \circ \mathbb{F} \circ \Omega(b) \cdot \partial_{y} F \circ \Omega(b) \| \\
\leq & \frac{C_{0} v^{n}}{1-\eta}\left\|\partial_{y} F \circ \Omega(a)-\partial_{y} F \circ \Omega(b)\right\|+C_{n} v^{n}\|\mathbb{F} \circ \Omega(b)-\mathbb{F} \circ \Omega(a)\| .
\end{aligned}
$$

We apply Lemma B.6 with $\mathcal{G}=\partial_{y} F, \tilde{D}=L_{1}, D_{0}=1, D^{\prime}=2 L_{1}$ and with $\mathcal{G}=F$ with $\tilde{D}=1, D_{0}=1, D^{\prime}=2$. Recalling (B.28), we obtain

$$
\left\|\partial_{s} \Phi(a)-\partial_{s} \Phi(b)\right\| \leq\left[\frac{C_{0} c_{0}}{(1-\eta) 4} L_{1}+\frac{C_{n} c_{0}}{4}\right]\|u\|^{\tau} .
$$

Next, by (B.15),

$$
\begin{aligned}
\left\|\partial_{S} F^{n}(a)-\partial_{s} F^{n}(b)\right\| \leq & \|\left[A \circ \mathbb{F} \cdot \partial_{y} F+B \circ \mathbb{F}\right] \circ \Omega(b) \\
& -\left[A \circ \mathbb{F} \cdot \partial_{y} F+B \circ \mathbb{F}\right] \circ \Omega(a)\|\cdot\| \partial_{s} \Phi \|_{\mathcal{C}^{0}} \\
& +\left\|\left[A \circ \mathbb{F} \cdot \partial_{y} F+B \circ \mathbb{F}\right] \circ \Omega\right\|_{\mathcal{C}^{0}} \cdot\left\|\partial_{s} \Phi(b)-\partial_{s} \Phi(a)\right\| .
\end{aligned}
$$

Using Lemma B.5 again,

$$
\begin{aligned}
& \left\|A \circ \mathbb{F} \circ \Omega(a) \cdot \partial_{y} F \circ \Omega(a)+B \circ \mathbb{F} \circ \Omega(a)-A \circ \mathbb{F} \circ \Omega(b) \cdot \partial_{y} F \circ \Omega(b)-B \circ \mathbb{F} \circ \Omega(b)\right\| \\
& \quad \leq\|A \circ \mathbb{F} \circ \Omega(a)\|\left\|\partial_{y} F \circ \Omega(a)-\partial_{y} F \circ \Omega(b)\right\|+C_{n}\|\mathbb{F} \circ \Omega(b)-\mathbb{F} \circ \Omega(a)\| \\
& \quad \leq C_{0} \lambda^{-n}\left\|\partial_{y} F \circ \Omega(a)-\partial_{y} F \circ \Omega(b)\right\|+C_{n} v^{n}\|\mathbb{F} \circ \Omega(b)-\mathbb{F} \circ \Omega(a)\| .
\end{aligned}
$$


Arguing as above and remembering (B.16), (B.8) and (B.9), we obtain, for all $n \in$ $\left\{n_{\star}, \ldots, 2 n_{\star}\right\}$,

$$
\left\|\partial_{S} F^{n}\right\|_{\mathcal{C}^{\tau}} \leq 1+\sigma_{1}^{n} C_{0}^{2} c_{0} L_{1}+c_{0} C_{n}<2 L_{1}
$$

provided

$$
L_{1}>\max \left\{1,2 c_{0} \max \left\{C_{n_{\star}}, \ldots, C_{2 n_{\star}}\right\}\right\} .
$$

For estimating the Hölder constant of $\partial_{s}^{\alpha} F^{n},|\alpha| \in\{2, \ldots, r-1\}$, we can use (B.19). (Since the argument uses a bound on $\partial_{S}^{\beta} F^{n}$ for $|\beta|=|\alpha|+1$, we stop at $|\alpha| \leq r-1$.) Indeed, recalling (B.16) and (B.18) and arguing similarly to before yields

$$
\begin{aligned}
\left\|\partial_{s}^{\alpha} F^{n}(a)-\partial_{s}^{\alpha} F^{n}(b)\right\| & \leq\left\{\left[\frac{C_{0}}{1-\eta} \theta^{n}\right]^{|\alpha|+2} L_{1}^{|\alpha|^{2}}+\sum_{j=0}^{|\alpha|-1} C_{\sharp} L_{1}^{(|\alpha|-1)^{2}-(j-1)^{2}} L_{1}^{j^{2}}\right\}\|u\|^{\tau} \\
& \leq\left\{\left[\frac{C_{0}}{1-\eta} \theta^{n}\right]^{|\alpha|+2}+C_{\sharp} L^{-2}\right\} L_{1}^{|\alpha|^{2}}\|u\|^{\tau} \leq\left(L_{1} / 2\right)^{|\alpha|^{2}}\|u\|^{\tau},
\end{aligned}
$$

provided $L_{1}$ has been chosen large enough. To obtain the estimate for all $n \in \mathbb{N}$, it suffices to write $k=k n_{\star}+m$, with $m \in\left\{n_{\star}, \ldots, 2 n_{\star}\right\}$, and then iterate the inequalities.

We are left with the study of $H^{F}$. Recalling (B.5), (B.6) and (B.15) and differentiating (B.13) with respect to $u$ yields

$$
\begin{aligned}
\partial_{u} \Phi & =-\left(E \circ \mathbb{F} \circ \Omega+C \circ \mathbb{F} \circ \Omega \cdot \partial_{y} F \circ \Omega\right)^{-1} C \circ \mathbb{F} \circ \Omega \cdot \partial_{x} F \circ \Omega \cdot D \Upsilon \\
\partial_{u} F^{n} & =\left[A \circ \mathbb{F}-\left(A \circ \mathbb{F} \cdot \partial_{y} F+B \circ \mathbb{F}\right)\left(E \circ \mathbb{F}+C \circ \mathbb{F} \cdot \partial_{y} F\right)^{-1} C \circ \mathbb{F}\right] \circ \Omega \cdot \partial_{x} F \circ \Omega \cdot D \Upsilon \\
& =\left[A \circ \mathbb{F} \circ \Omega-\partial_{s} F^{n} \cdot C \circ \mathbb{F} \circ \Omega\right] \cdot \partial_{x} F \circ \Omega \cdot D \Upsilon=:\left[\Lambda \cdot \partial_{x} F \circ \Omega\right] \cdot D \Upsilon .
\end{aligned}
$$

We can now compute

$$
\begin{aligned}
H_{l}^{F^{n}} \circ\left(\mathbb{F}^{n}\right)^{-1} & =\sum_{i} \partial_{u_{i}}\left[\left(\partial_{s_{l}} F_{i}^{n}\right) \circ\left(\mathbb{F}^{n}\right)^{-1}\right]=\sum_{i, k}\left[\partial_{s_{l}} \partial_{u_{k}} F_{i}^{n} \cdot\left(\partial_{u} F^{n}\right)_{k, i}^{-1}\right] \circ\left(\mathbb{F}^{n}\right)^{-1} \\
& =\operatorname{Trace}\left(\left[\partial_{s_{l}} \partial_{u} F^{n}\right]\left(\partial_{u} F^{n}\right)^{-1}\right) \circ\left(\mathbb{F}^{n}\right)^{-1} .
\end{aligned}
$$

Thus, using (B.35),

$$
\begin{aligned}
H_{l}^{F^{n}=} & \operatorname{Trace}\left[\left(\partial_{s_{l}} \Lambda\right) \cdot \partial_{x} F \circ \Omega \cdot D \Upsilon\left(\partial_{u} F^{n}\right)^{-1}\right. \\
& \left.+\Lambda \cdot\left(\partial_{s_{l}}\left\{\partial_{x} F \circ \Omega\right\}\right) \cdot D \Upsilon\left(\partial_{u} F^{n}\right)^{-1}\right] \\
= & \operatorname{Trace}\left[\left(\partial_{s_{l}} \Lambda\right) \Lambda^{-1}\right]+\sum_{k} \partial_{s_{l}} \Phi_{k}\left\{\operatorname{Trace}\left[\left(\partial_{y_{k}} \partial_{x} F\left(\partial_{x} F\right)^{-1}\right]\right\} \circ \Omega\right. \\
= & H^{F} \circ \Omega \cdot \partial_{s_{l}} \Phi+\operatorname{Trace}\left[\left(\partial_{s} \Lambda\right) \Lambda^{-1}\right] .
\end{aligned}
$$

Moreover, note that Trace $\left[\left(\partial_{S_{l}} \Lambda\right) \Lambda^{-1}\right](0)=\sum_{k}\left\{\operatorname{Trace}\left[\partial_{y_{k}} A \cdot A^{-1}\right] \cdot\left[E^{-1}\right]_{k l}\right\}(0)$. Hence, using (B.16), we obtain, for all $n \in\left\{n_{\star}, \ldots, 2 n_{\star}\right\}$,

$$
\left\|H^{F^{n}}\right\|_{\mathcal{C}^{0}} \leq\left\|H^{F} \cdot \partial_{S_{l}} \Phi\right\|_{\mathcal{C}^{0}}+c_{n} \leq \frac{C_{0}}{1-\eta} v^{n}\left\|H^{F}\right\|_{\mathcal{C}^{0}}+c_{n} \leq L_{1} / 2,
$$


provided $L_{1}$ is large enough. Differentiating (B.36) yields, for each $0<l \leq r-2$,

$$
\begin{gathered}
\partial_{s_{j_{l}}} \cdots \partial_{s_{j_{1}}} H^{F^{n}}=\sum_{i_{1}, \ldots, i_{l}}\left[\partial_{y_{i_{l}}} \cdots \partial_{y_{i_{1}}} H^{F}\right] \circ \Omega \cdot \partial_{s} \Phi \cdot \partial_{s_{j_{1}}} \Phi_{s_{i_{1}}} \cdots \partial_{s_{j_{l}}} \Phi_{i_{l}} \\
+\bar{\Theta}_{l}\left(D S \circ \mathbb{F} \circ \Omega, \ldots, D^{l+2} S \circ \mathbb{F} \circ \Omega, \partial_{s}^{l+1} F^{n}, \ldots, \partial_{s} F^{n}, \partial_{y}^{l} F \circ \Omega, \ldots\right. \\
\left.\ldots \partial_{y} F \circ \Omega, H^{F} \circ \Omega, \ldots,\left[\partial_{y}^{l-1} H^{F}\right] \circ \Omega\right),
\end{gathered}
$$

where $\bar{\Theta}_{l}$ is a sum of terms that either do not depend on $\partial_{s}^{p} H^{F^{n}}$, for all $p<l$, or are linear in a $\partial_{s}^{p} H^{F^{n}}$, for some $p<l, k_{p, j}$-multilinear in $\partial_{y}^{j} F$, for $j \in\{2, \ldots, l+1\}$, and $q_{p, j}$ multilinear in $\partial_{s}^{j} F^{n}$, for $j \in\{2, \ldots, l+2\}$, such that (we use the convention that $q_{p, l+2}=0$ and $\left.\partial_{s}^{-1} H^{F^{n}}=1\right)$

$$
\sup _{p \in\{-1, \ldots l-1\}}\left[p+\sum_{j=2}^{l+2}\left(k_{p, j}+q_{p, j}\right)(j-1)\right] \leq l .
$$

Let us verify this. Equation (B.36) shows that it is true for $l=0$. Let us assume that it true for $l-1$. Then, by differentiating the first term, we obtain the correct term linear in $\partial^{l} H^{F}$. The other terms are linear in $\partial^{l-1} H^{F}$ and linear in $\partial^{2} F \circ \Omega$ (see equation (B.15)) and hence $p^{\prime}=l-1, k_{l-1,2}=1$ and all the other degrees are zero, so $p^{\prime}+k_{l-1,2} \leq l$. Differentiating $\bar{\Theta}_{l-1}$ with respect to $D^{m} S \circ \mathbb{F} \circ \Omega$ does not change the multilinearity indices. Differentiating with respect to $\partial_{s}^{j} F^{n}$ yields, for each $p$, a term with $p^{\prime}=p$, $q_{p^{\prime}, j}^{\prime}=q_{p, j}-1$ multilinear in $\partial_{s}^{j} F^{n}$ and $q_{p^{\prime}, j+1}^{\prime}=q_{p, j+1}+1$ multilinear in $\partial_{s}^{j+1} F^{n}$. Thus $p^{\prime}+\sum_{j=2}^{l+2}\left(k_{p^{\prime}, j}^{\prime}+q_{p^{\prime}, j}^{\prime}\right)(j-1) \leq l$. The same happens if one differentiates with respect to $\partial_{y}^{j} F \circ \Omega$ for $j \geq 2$. On the other hand, differentiating with respect to $\partial_{y} F \circ \Omega$ yields a term in which $p^{\prime}=p, k_{p^{\prime}, 2^{\prime}}=k_{p, 2}+1$, and thus $p^{\prime}+\sum_{j=2}^{l+2}\left(k_{p^{\prime}, j}^{\prime}+q_{p^{\prime}, j}^{\prime}\right)(j-$ $1) \leq l$. Finally, if we differentiate with respect to $\partial_{y}^{j} H^{F} \circ \Omega$ for $0 \leq j<l-1$, we have a term with $p^{\prime}=p+1$ and $k_{p^{\prime}, j}^{\prime}=k_{p, j}, q_{p^{\prime}, j}^{\prime}=q_{p, j}$, and thus, again, $p^{\prime}+\sum_{j=2}^{l+2}\left(k_{p^{\prime}, j}^{\prime}+\right.$ $\left.q_{p^{\prime}, j}^{\prime}\right)(j-1) \leq l$, which proves the claim.

Remembering (B.16), definition (B.2) and equation (B.21), it follows that, for all $l \in$ $\{1, \ldots, r-2\}$,

$$
\begin{aligned}
\left\|\partial_{s}^{l} H^{F^{n}}\right\| & \leq 8^{-r(l+1)} L_{1}^{(l+1)^{2}}+\sup _{p \in\{-1, \ldots, l-1\}} C_{\sharp} L_{1}^{(p+1)^{2}+\sum_{j=2}^{l+2}\left(k_{p, j}+q_{p, j}\right)(j-1)^{2}} \\
& \leq 8^{-(l+1)^{2}} L_{1}^{(l+1)^{2}}+\sup _{p \in\{-1, \ldots, l-1\}} C_{\sharp} L_{1}^{(p+1)^{2}+\left[\sum_{j=2}^{l+2}\left(k_{p, j}+q_{p, j}\right)(j-1)\right](l+1)} \\
& \leq 8^{-(l+1)^{2}} L_{1}^{(l+1)^{2}}+\sup _{p \in\{-1, \ldots, l-1\}} C_{\sharp} L_{1}^{(p+1)^{2}+(l-p)(l+1)} \\
& \leq\left[8^{-(l+1)^{2}}-C_{\sharp} L_{1}^{-l}\right] L_{1}^{(l+1)^{2}} \leq\left(\frac{L_{1}}{2}\right)^{(l+1)^{2}}
\end{aligned}
$$

provided $L_{1}$ is chosen large enough. 
To prove the bound on the Hölder semi-norm, we use (B.38) and (B.31) and proceed as in (B.34). For each $l \leq r-3$,

$$
\begin{aligned}
\left\|\partial_{s}^{l} H^{F^{n}}(a)-\partial_{s}^{l} H^{F^{n}}(b)\right\| \leq & \left\|\partial_{y}^{l} H^{F} \circ \Omega(a)-\partial_{y}^{l} H^{F} \circ \Omega(b)\right\| C_{\star}^{l+1} v^{(l+1) n} \\
& +C_{\sharp} L_{1}^{(l+1)^{2}+1}\|u\|^{\tau_{0}}+\left\|\bar{\Theta}_{l}(a)-\bar{\Theta}_{l}(b)\right\|,
\end{aligned}
$$

where we have used (B.16), (B.31) and (B.32). Next, we use Lemma B.6, with $\mathcal{G}=\partial_{y}^{l} H^{F}$, $D_{0}=L_{1}^{(l+1)^{2}}, D^{\prime}=\tilde{D}=L_{1}^{(l+2)^{2}}$ and $\tau=\tau_{0}$, to write

$$
\begin{aligned}
\left\|\partial_{s}^{l} H^{F^{n}}(a)-\partial_{s}^{l} H^{F^{n}}(b)\right\| \leq & C_{l} \nu^{l n} L_{1}^{(l+2)^{2}}\|u\|^{\tau_{0}} \\
& +C_{\sharp} L_{1}^{(l+1)^{2}+1}\|u\|^{\tau_{0}}+\left\|\bar{\Theta}_{l}(a)-\bar{\Theta}_{l}(b)\right\| .
\end{aligned}
$$

The claim then follows by induction and using the known structure of $\bar{\Theta}_{l}$.

We conclude the section by clarifying the relationship between the function $H^{F}$ and the holonomy associated with the foliation $\mathbb{F}$. The next lemma shows that the Jacobian of the holonomy can be seen as a flow of which $H^{F}$ is the 'generator'.

LEMMA B.7. If $W \in \mathcal{W}_{L}^{r, 0}$, then there exists $C>0$ and $\rho_{0}>0$ such that, for each $\xi \in M$, $0<\rho<\rho_{0}$ and $\left\|\left(x^{\prime}, y^{\prime}\right)\right\| \leq \rho$, we have $\left\|\operatorname{det}\left(\partial_{x} F_{\xi}\right)\left(x^{\prime}, \cdot\right)\right\|_{\mathcal{C}^{q}} \leq C$. More precisely, setting $J_{\xi}^{F}(x, y)=\operatorname{det}\left(\partial_{x} F_{\xi}\right)(x, y)$, we have

$$
\begin{aligned}
& \partial_{y} J_{\xi}^{F}=J_{\xi}^{F} \cdot H_{\xi}^{F} \circ \mathbb{F}, \\
& J_{\xi}^{F}(x, 0)=1 .
\end{aligned}
$$

Proof. Let $\left(x^{\prime}, y^{\prime}\right)$ be as in the lemma's assumption. First, note that, for each vector $e_{i} \in$ $\mathbb{R}^{d}$,

$$
\begin{aligned}
\partial_{y_{i}} \operatorname{det}\left(\partial_{x} F_{\xi}\right)\left(x^{\prime}, y^{\prime}\right) & =\operatorname{det}\left(\partial_{x} F_{\xi}\right) \lim _{h \rightarrow 0} \frac{\operatorname{det}\left(\left(\partial_{x} F_{\xi}\right)\left(x^{\prime}, y^{\prime}\right)^{-1} \cdot \partial_{x} F_{\xi}\left(x^{\prime}, y^{\prime}+h e_{i}\right)\right)-1}{h} \\
& =\operatorname{det}\left(\partial_{x} F_{\xi}\right) \lim _{h \rightarrow 0} \frac{e^{\operatorname{Trace}\left(\ln \left(\mathbb{1}+\left(\partial_{x} F_{\xi}\right)\left(x^{\prime}, y^{\prime}\right)^{-1} \partial_{y_{i}} \partial_{x} F_{\xi}\left(x^{\prime}, y^{\prime}\right) h\right)\right)}-1}{h} \\
& =\operatorname{det}\left(\partial_{x} F_{\xi}\right)\left(x^{\prime}, y^{\prime}\right) \operatorname{Trace}\left(\left(\partial_{x} F_{\xi}\right)\left(x^{\prime}, y^{\prime}\right)^{-1} \partial_{x}\left(\partial_{y_{i}} F_{\xi}\right)\left(x^{\prime}, y^{\prime}\right)\right) .
\end{aligned}
$$

Thus

$$
\partial_{y} \operatorname{det}\left(\partial_{x} F_{\xi}\right)\left(x^{\prime}, y^{\prime}\right)=\operatorname{det}\left(\partial_{x} F_{\xi}\right)\left(x^{\prime}, y^{\prime}\right) \cdot H_{\xi}^{F} \circ \mathbb{F}\left(x^{\prime}, y^{\prime}\right),
$$

which immediately implies the lemma since $\operatorname{det}\left(\partial_{x} F_{\xi}\right)\left(x^{\prime}, 0\right)=1$ by construction.

Remark B.8. Lemma B.7 implies that, for each measurable set $B \subset \mathbb{R}^{d_{u}}$ and $|\beta| \leq r-1$,

$$
\left|\partial_{y}^{\beta}\right| F(B, y)||=\left|\partial_{y}^{\beta} \int_{F(B, y)} d x\right|=\left|\int_{B} \partial_{y}^{\beta} \operatorname{det}\left(\partial_{x} F\right)(x, y) d x\right| \leq C|B| .
$$

Note that the first and last term of the above inequality do not involve $\partial_{x} F$, and hence it holds also for $F$ non-differentiable with respect to $x$, provided they are the limits of foliations $F_{k}$ (in the sense that the $\partial_{y}^{\beta} F_{k}$ converge) that satisfy the inequality uniformly. The same remark holds also for equation (B.40). In other words, if we consider the true 
invariant foliation, where $\partial_{x} F$ may make no sense, $H^{F}$ is still well defined (see Remark B.3 for details), and so, by (B.40), is the Jacobian of the holonomy $J^{F}$.

\section{Appendix. Test functions}

Proof of Lemma 2.15. By (B.13), it follows that

$$
\begin{aligned}
\varphi \circ T^{n} \circ \phi_{i}^{-1} \circ \mathbb{F}^{n}(u, s) & =\varphi \circ \phi_{j}^{-1} \circ S^{-1} \circ \mathbb{F}^{n}(u, s)=\varphi \circ \phi_{j}^{-1} \circ \mathbb{F}(\Omega(u, s)) \\
& =\varphi \circ \phi_{j}^{-1} \circ \mathbb{F}(\Upsilon(u), \Phi(u, s)) .
\end{aligned}
$$

Accordingly, $\left\|\varphi \circ T^{n}\right\|_{\mathcal{C}^{0}}^{T^{-n} W} \leq\|\varphi\|_{\mathcal{C}^{0}}^{W}$ and

$$
\partial_{s}\left[\varphi \circ T^{n} \circ \phi_{i}^{-1} \circ \mathbb{F}^{n}\right](u, s)=\sum_{l} \partial_{z_{l}}\left[\varphi \circ \phi_{j}^{-1} \circ \mathbb{F}\right](\Omega(u, s)) \partial_{s} \Phi_{l}(u, s) .
$$

Then, by differentiating further, the above computation yields, for some $C_{*}>0$,

$$
\varpi\left|\partial_{s}^{q}\left[\varphi \circ T^{n} \circ \phi_{i}^{-1} \circ \mathbb{F}^{n}\right](u, s)\right| \leq\|\varphi\|_{q}^{W}\left\|\partial_{s} \Phi_{l}\right\|_{\mathcal{C}^{0}}^{q}+\varpi^{-1} C_{*}\|\varphi\|_{q-1}^{W} .
$$

By (B.16), $\left\|\partial_{s} \Phi\right\|_{\mathcal{C}^{0}} \leq C_{\sharp} \sigma^{n}$, while there exists $A_{0}>1$ such that $\left\|\partial_{s}^{i} \Phi\right\|_{\mathcal{C}^{0}} \leq A_{0}$ for all $i \leq r$.

From this and recalling the definition (2.7), it follows that

$$
\left\|\varphi \circ T^{n}\right\|_{q}^{T^{-n} W} \leq A_{0}\|\varphi\|_{q}^{W} .
$$

On the other hand, recalling (2.8), there exists $B_{0}>0$ such that

$$
\begin{aligned}
\left\|\varphi \circ T^{n}\right\|_{q+1}^{T^{-n} W} & =\left\|\partial_{s} \varphi \circ T^{n}\right\|_{q}^{T^{-n} W}+\varpi^{q+1}\left\|\varphi \circ T^{n}\right\|_{C^{0}}^{T^{-n} W} \\
& \leq A_{0} \sigma^{q n}\|\varphi\|_{q+1}^{W}+\left(1+\varpi^{-1} C_{*}\right)\|\varphi\|_{\mathcal{C}^{q}}^{W} \\
& \leq A_{0} \sigma^{q n}\|\varphi\|_{q+1}^{W}+B_{0}\|\varphi\|_{\mathcal{C}^{q}}^{W} .
\end{aligned}
$$

\section{REFERENCES}

[AGT] A. Avila, S. Gouëzel and M. Tsujii. Smoothness of solenoidal attractors. Discrete Contin. Dyn. Syst. 15(1) (2006), 21-35.

[B1] V. Baladi. Anisotropic Sobolev spaces and dynamical transfer operators: $\mathcal{C}^{\infty}$ foliations. Algebraic and Topological Dynamics (Contemporary Mathematics, 385). American Mathematical Society, Providence, RI, 2005, pp. 123-135.

[B2] V. Baladi. The quest for the ultimate anisotropic Banach space. J. Stat. Phys. 166(3-4) (2017), 525-557.

[B3] V. Baladi. Characteristic functions as bounded multipliers on anisotropic spaces. Proc. Amer. Math. Soc. 146(10) (2018), 4405-4420.

[B4] V. Baladi. Dynamical Zeta Functions and Dynamical Determinants for Hyperbolic Maps (Ergebnisse der Mathematik und ihrer Grenzgebiete. 3. Folge/A Series of Modern Surveys in Mathematics, 68). Springer International, New York, 2018.

[B5] V. Baladi. Dynamical Zeta Functions and Dynamical Determinants for Hyperbolic Maps. A Functional Approach (A Series of Modern Surveys in Mathematics, 68). Springer, Cham, 2018.

[BaL] V. Baladi and C. Liverani. Exponential decay of correlations for piecewise cone hyperbolic contact flows. Comm. Math. Phys. 314(3) (2012), 689-773.

[BDL] V. Baladi, M. F. Demers and C. Liverani. Exponential decay of correlations for finite horizon Sinai billiard flows. Invent. Math. 211(1) (2018), 39-177.

[BG1] V. Baladi and S. Gouëzel. Good Banach spaces for piecewise hyperbolic maps via interpolation. Ann. Inst. H. Poincaré Anal. Non Linéaire 26(4) (2009), 1453-1481. 
[BG2] V. Baladi and S. Gouëzel. Banach spaces for piecewise cone-hyperbolic maps. J. Mod. Dyn. 4(1) (2010), 91-137.

[BKL] M. Blank, G. Keller and C. Liverani. Ruelle-Perron-Frobenius spectrum for Anosov maps. Nonlinearity 15(6) (2001), 1905-1973.

[BT] V. Baladi and M. Tsujii. Anisotropic Hölder and Sobolev spaces for hyperbolic diffeomorphisms. Ann. Inst. Fourier 57 (2007), 127-154.

[BT1] V. Baladi and M. Tsujii. Dynamical determinants and spectrum for hyperbolic diffeomorphisms. Geometric and Probabilistic Structures in Dynamics (Contemporary Mathematics, 469). Ed. K. Burns, D. Dolgopyat and Y. Pesin. American Mathematical Society, Providence, RI, 2008, pp. 29-68. Volume in honour of M. Brin's 60th birthday.

[Bu13] O. Butterley. An alternative approach to generalised BV and the application to expanding interval maps. Discrete Contin. Dyn. Syst. 33(8) (2013), 3355-3363.

[BuL] O. Butterley and C. Liverani. Smooth Anosov flows: correlation spectra and stability. J. Mod. Dyn. 1 (2007), 301-322.

[BuL2] O. Butterley and C. Liverani. Robustly invariant sets in fiber contracting bundle flows. J. Mod. Dyn. 7(2) (2013), 255-267.

[D17] A. Drouot. Stochastic stability of Pollicott-Ruelle resonances. Comm. Math. Phys. 356(2) (2017), 357-396.

[DL] M. F. Demers and C. Liverani. Stability of statistical properties in two-dimensional piecewise hyperbolic maps. Trans. Amer. Math. Soc. 360(9) (2008), 4777-4814.

[DyZ] S. Dyatlov and M. Zworski. Dynamical zeta functions for Anosov flows via microlocal analysis. Ann. Sci. Éc. Norm. Supér. 49(3) (2016), 543-577.

[DZ1] M. F. Demers and H.-K. Zhang. Spectral analysis of hyperbolic systems with singularities. Nonlinearity 27(3) (2014), 379-433.

[DZ2] M. F. Demers and H.-K. Zhang. A functional analytic approach to perturbations of the Lorentz gas. Comm. Math. Phys. 324(3) (2013), 767-830.

[DZ3] M. F. Demers and H.-K. Zhang. Spectral analysis of the transfer operator for the Lorentz gas. J. Mod. Dyn. 5(4) (2011), 665-709.

[F11] F. Faure. Semiclassical origin of the spectral gap for transfer operators of a partially expanding map. Nonlinearity 24(5) (2011), 1473-1498.

[FR] F. Faure and N. Roy. Ruelle-Pollicott resonances for real analytic hyperbolic maps. Nonlinearity 19(6) (2006), 1233-1252.

[FRS] F. Faure, N. Roy and J. Sjöstrand. Semi-classical approach for Anosov diffeomorphisms and Ruelle resonances. Open Math. J. 1 (2008), 35-81.

[FT1] F. Faure and M. Tsujii. Semiclassical approach for the Ruelle-Pollicott spectrum of hyperbolic dynamics. Analytic and Probabilistic Approaches to Dynamics in Negative Curvature (Springer INdAM Series, 9). Springer, Cham, 2014, pp. 65-135.

[FT2] F. Faure and M. Tsujii. The semiclassical zeta function for geodesic flows on negatively curved manifolds. Invent. Math. 208(3) (2017), 851-998.

[G10] S. Gouëzel. Almost sure invariance principle for dynamical systems by spectral methods. Ann. Probab. 38(4) (2010), 1639-1671.

[G15] S. Gouëzel. Limit theorems in dynamical systems using the spectral method. Hyperbolic Dynamics, Fluctuations and Large Deviations (Proceedings of Symposia in Pure Mathematics, 89). American Mathematical Society, Providence, RI, 2015, pp. 161-193.

[Ga18] S. Galatolo. Quantitative statistical stability, speed of convergence to equilibrium and partially hyperbolic skew products. J. Éc. Polytech. Math. 5 (2018), 377-405

[GL] S. Gouëzel and C. Liverani. Banach spaces adapted to Anosov systems. Ergod. Th. \& Dynam. Sys. 26(1) (2006), 189-217.

[GL1] S. Gouëzel and C. Liverani. Compact locally maximal hyperbolic sets for smooth maps: fine statistical properties. J. Differential Geom. 79(3) (2008), 433-477.

[GL18] S. Galatolo and R. Lucena. Spectral gap and quantitative statistical stability for systems with contracting fibers and Lorenz-like maps. Discrete Contin. Dyn. Syst. 40(3) (2020), 309-1360.

[GL19] P. Giulietti and C. Liverani. Parabolic dynamics and anisotropic Banach spaces. J. Eur. Math. Soc. (JEMS) 21(9) (2019), 2793-2858.

[GLP] P. Giulietti, C. Liverani and M. Pollicott. Anosov flows and dynamical zeta functions. Ann. of Math. 178(2) (2013), 687-773.

[He] H. Hennion. Sur un théorème spectral et son application aux noyaux Lipchitziens. Proc. Amer. Math. Soc. 118 (1993), 627-634.

[HW] B. Hasselblatt and A. Wilkinson. Prevalence of non-Lipschitz Anosov foliations. Ergod. Th. \& Dynam. Sys. 19(3) (1999), 643-656. 
[J] J.-L. Journé. A regularity lemma for functions of several variables. Rev. Mat. Iberoam. 4(2) (1988), 187-193.

[KH] A. Katok and B. Hasselblatt. Introduction to the Modern Theory of Dynamical Systems (Encyclopedia of Mathematics and its Applications, 54). Cambridge University Press, Cambridge, 1995. With a supplementary chapter by Katok and Leonardo Mendoza.

[KL1] G. Keller and C. Liverani. Stability of the spectrum for transfer operators. Ann. Sc. Norm. Super. Pisa Cl. Sci. (4) 28(1) (1999), 141-152.

[KL2] G. Keller and C. Liverani. Uniqueness of the SRB measure for piecewise expanding weakly coupled map lattices in any dimension. Comm. Math. Phys. 262(1) (2006), 33-50.

[KL3] G. Keller and C. Liverani. Rare events, escape rates and quasistationarity: some exact formulae. $J$. Stat. Phys. 135(3) (2009), 519-534.

[KL4] G. Keller and C. Liverani. Map lattices coupled by collisions. Comm. Math. Phys. 291(2) (2009), 591-597.

[KS] A. Katok, J.-M. Strelcyn, F. Ledrappier and F. Przytycki. Invariant Manifolds, Entropy and Billiards; Smooth Maps with Singularities (Lecture Notes in Mathematics, 1222). Springer-Verlag, Berlin, 1986, pp. viii +283 .

[L] C. Liverani. On contact Anosov flows. Ann. of Math. 159(3) (2004), 1275-1312.

[Li13a] C. Liverani. A footnote on expanding maps. Discrete Contin. Dyn. Syst. 33(8) (2013), 3741-3751.

[Li13b] C. Liverani. Multidimensional expanding maps with singularities: a pedestrian approach. Ergod. Th. \& Dynam. Sys. 33(1) (2013), 168-182.

[Liv] C. Liverani. Rigorous numerical investigation of the statistical properties of piecewise expanding maps. A feasibility study. Nonlinearity 14(3) (2001), 463-490.

[Nu] R. D. Nussbaum. The radius of the essential spectrum. Duke Math. J. 37(3) (1970), 473-478.

[PS] Y. B. Pesin and Y. G. Sinai. Space-time chaos in the system of weakly interacting hyperbolic systems. J. Geom. Phys. 5(3) (1988), 483-492.

[PSW] C. Pugh, M. Shub and A. Wilkinson. Hölder foliations. Duke Math. J. 86(3) (1997), 517-546.

[Sa00] B. Saussol. Absolutely continuous invariant measures for multidimensional expanding maps. Israel J. Math. 116 (2000), 223-248.

[T1] M. Tsujii. Decay of correlations in suspension semi-flows of angle multiplying maps. Ergod. Th. \& Dynam. Sys. 28(1) (2008), 291-317.

[T2] M. Tsujii. Quasi-compactness of transfer operators for contact Anosov flows. Nonlinearity 23(7) (2010), 1495-1545. 